\title{
Spectral Analysis of a Stochastic Ising Model in Continuum
}

\author{
Yu. Kondratiev • E. Zhizhina
}

Received: 16 September 2006 / Accepted: 9 June 2007 / Published online: 25 July 2007

(C) Springer Science+Business Media, LLC 2007

\begin{abstract}
We consider an equilibrium stochastic dynamics of spatial spin systems in $\mathbb{R}^{d}$ involving both a birth-and-death dynamics and a spin flip dynamics as well. Using a general approach to the spectral analysis of corresponding Markov generator, we estimate the spectral gap and construct one-particle invariant subspaces for the generator.
\end{abstract}

Keywords Birth-and-death process · Continuous system · Gibbs measure · Glauber dynamics $\cdot$ Continuous Ising model

\section{Introduction}

In this paper we study an equilibrium stochastic dynamics of continuous spin systems involving a birth-and-death process as well as a spin flip dynamics. The dynamics is a natural generalization of the stochastic Ising model and a Glauber-type dynamics of continuous gas which has been under consideration in $[2,4,6]$. The generator of this dynamics is a selfadjoint operator in $L^{2}$-space w.r.t. an equilibrium measure. The main goal of the present paper is to study the structure of the low-lying spectrum of the infinite volume dynamics generator: to estimate the spectral gap, to construct leading invariant subspaces of the

Dedicated to our admired teacher and friend Robert Minlos on occasion of his 75th birthday.

The financial support of SFB-701, Bielefeld University, is gratefully acknowledged. The work is partially supported by RFBR grant 05-01-00449, Scientific School grant 934.2003.1, CRDF grant RUM1-2693-MO-05.

Yu. Kondratiev (凶)

Dept. of Mathematics and BiBoS, Bielefeld University, Bielefeld, Germany

e-mail: kondrat@math.uni-bielefeld.de

Yu. Kondratiev

NaUKMA, Kiev, Ukraine

E. Zhizhina

IITP, Russian Acad. Sci., Moscow, Russia

e-mail: ejj@iitp.ru 
generator and to find the location of the corresponding isolated branches of the generator spectrum. We prove that involving in the dynamics a new spin flip action does not change essentially the structure of the low-lying spectrum of the generator if the intensity of spin flips is small enough. When the intensity of spin flips is increasing, the first spectral gap (a gap between 0 and an one-particle branch of the spectrum $\sigma_{1}$ ) is still preserved while the second gap (a gap between an one particle branch $\sigma_{1}$ and the rest of the spectrum $\sigma_{2}$ ) could vanish. That means that we can estimate from above and from below the decay of autocorrelation functions when the intensity of the spin flip is small enough, but for large values of the intensity we have only the upper bound. We use here general approaches from [1, 3, $8,9,14]$ to the spectral analysis of the generators of stochastic dynamics systems together with modifications of methods developed in $[4,5]$ for the study of spatial dynamics.

Theorem 2 contains the main result of this paper on the existence of an one-particle invariant subspace of the generator and the corresponding isolated one-particle branch of the spectrum in a low activity - high temperature regime when the spin flip intensity is small enough. We also show-it is a statement of Theorem 1-that for any value of the spin flip intensity an infinite volume dynamics generator has a spectral gap, and we found a lower estimate on the spectral gap. We note that a lower estimate on the spectral gap has been also found using other technique (for instance, coercitivity identity), see [6,13]. We exploit here an approach which is also applicable for the separation of low-lying branches of the generator spectrum. One of the goal of the paper is to show the universality of the general scheme of the spectral analysis of infinite-particle operators developed in numerous papers of R.A. Minlos and his collaborators, see for instance [1, 3, 4, 7-9].

\section{Glauber Dynamics for Continuous Multi-Component Models}

\subsection{Glauber Dynamics of Continuous Gas}

In this section we shortly remind main constructions of a Glauber-type dynamics of continuous gas. The configuration space $\Gamma:=\Gamma\left(\mathbb{R}^{d}\right)$ is the set of all locally finite subsets of $\mathbb{R}^{d}$. We consider in the space $\Gamma$ a topology with respect to which all maps $\Gamma \ni \gamma \mapsto\langle f, \gamma\rangle:=$ $\sum_{x \in \gamma} f(x), f \in \mathcal{D}$, are continuous (here, $\mathcal{D}:=C_{0}^{\infty}\left(\mathbb{R}^{d}\right)$ is the space of all infinitely differentiable real-valued functions on $\mathbb{R}^{d}$ with compact support). We will denote by $\mathcal{B}(\Gamma)$ the Borel $\sigma$-algebra on $\Gamma$ generated by this topology. Then the Poisson measures $\pi_{z}$ with activity $z, z>0$, are defined on $(\Gamma, \mathcal{B}(\Gamma))$ by the following properties:

(1) For any family of mutually disjoint bounded measurable domains $\Lambda_{1}, \ldots, \Lambda_{k}, \Lambda_{j} \subset \mathbb{R}^{d}$ random variables

$$
N_{\Lambda_{j}}(\gamma)=\left|\gamma_{\Lambda_{j}}\right|, \quad j=1, \ldots, k
$$

are independent. Here $|A|$ denotes the cardinality of a set $A, \gamma_{\Lambda}=\gamma \cap \Lambda$.

(2) Each random variable $N_{\Lambda_{j}}$ has the Poisson distribution

$$
\operatorname{Pr}\left(N_{\Lambda_{j}}(\gamma)=n\right)=\frac{z^{n}\left|\Lambda_{j}\right|^{n}}{n !} e^{-z\left|\Lambda_{j}\right|}, \quad j=1, \ldots, k,
$$

where $\left|\Lambda_{j}\right|$ is the volume of $\Lambda_{j}$ in $\mathbb{R}^{d},[10]$.

In other words, $\pi_{z}$ is the Poisson white noise measure on $\Gamma$ corresponding to the intensity measure $z d x$ on $\mathbb{R}^{d}$. 
The Gibbs reconstruction of the Poisson measure $\pi_{z}$ is defined by a formal Hamiltonian

$$
U(\gamma)=\sum_{x, y \in \gamma} \phi(x-y)
$$

and the inverse temperature $\beta>0$. Here and later on the sum is taken over unordered pairs of points $x, y \in \gamma$. The Gibbs measure $\mu_{\beta, z}$ is constructed as a limit when $\Lambda \nearrow \mathbb{R}^{d}$ of finite volume Gibbs measures corresponding to empty boundary conditions:

$$
\mu_{\beta, z}=\lim _{\Lambda \nearrow \mathbb{R}^{d}} \mu_{\beta, z}^{\Lambda} .
$$

The measure $\mu_{\beta, z}^{\Lambda}$ is defined by the following density w.r.t. the Poisson measure:

$$
\frac{d \mu_{\beta, z}^{\Lambda}}{d \pi_{z}}=\frac{1}{Z_{\Lambda}} \exp \left\{-\beta U\left(\gamma_{\Lambda}\right)\right\}, \quad \Lambda \subset \mathbb{R}^{d},
$$

where $Z_{\Lambda}$ is the normalizing factor. We will use below general assumptions on the pair potential $\phi(u)$ and on the parameters $\beta, z$ guaranteeing the existence of the limit (2.1), see for instance [11].

We will consider next a stationary Markov process on the state space $\Gamma$ with the invariant measure $\mu_{\beta, z}$. The generator of the corresponding stochastic semigroup in the functional space $L^{2}\left(\Gamma, \mu_{\beta, z}\right)$ has following form:

$$
(H F)(\gamma)=\sum_{x \in \gamma}(F(\gamma \backslash x)-F(\gamma))+z \int_{\mathbb{R}^{d}} e^{-\beta E(x, \gamma)}(F(\gamma \cup x)-F(\gamma)) d x,
$$

where $E(x, \gamma)$ is the relative energy of interaction between a particle located at $x$ and the configuration $\gamma$ :

$$
E(x, \gamma):= \begin{cases}\sum_{y \in \gamma} \phi(x-y), & \text { if } \sum_{y \in \gamma}|\phi(x-y)|<\infty \\ +\infty, & \text { otherwise. }\end{cases}
$$

This generator is associated with a Dirichlet form

$$
(-H F, F)=\mathcal{E}(F, F)=\int_{\Gamma} \sum_{x \in \gamma}|F(\gamma \backslash x)-F(\gamma)|^{2} d \mu_{\beta, z}(\gamma) .
$$

As it was shown in [6], under general conditions on the potential $\phi$ and the parameters $\beta, z$, there exists a stationary Markov process $\{\gamma(t), t \in \mathbb{R}\}$ on $\Gamma$ with the stationary measure $\mu_{\beta, z}$, such that the generator (2.4) of the process can be extended to a self-adjoint operator in $L^{2}\left(\Gamma, \mu_{\beta, z}\right)$, what is equivalent to the reversibility of the process $\gamma(t)$. This process is called the equilibrium Glauber dynamics which corresponds to the Gibbs measure $\mu_{\beta, z}$.

\subsection{Glauber Dynamics of Continuous Potts Models}

In this section we extend the above definition and constructions to the case of twocomponent continuous systems. The configuration space is the product of two configuration spaces associated with each component of the system:

$$
\gamma=\left(\gamma_{+}, \gamma_{-}\right) \in \Gamma_{+} \times \Gamma_{-} .
$$


Both $\Gamma_{+}$and $\Gamma_{-}$are defined as above in Sect. 2.1, and we can consider the product of Poisson measures $\pi_{z}^{(+)} \times \pi_{z}^{(-)}$. A Gibbs measure for considered two-component system is formally defined as follows

$$
d \mu_{\beta, z}\left(\gamma_{+}, \gamma_{-}\right)=\frac{1}{Z} \exp \left\{-\beta U\left(\gamma_{+}, \gamma_{-}\right)\right\} d \pi_{z}^{(+)}\left(\gamma_{+}\right) d \pi_{z}^{(-)}\left(\gamma_{-}\right)
$$

with a Hamiltonian

$$
U\left(\gamma_{+}, \gamma_{-}\right)=\sum_{x \in \gamma_{+}, y \in \gamma_{+}} \phi_{+}(x-y)+\sum_{x \in \gamma_{-}, y \in \gamma_{-}} \phi_{-}(x-y)+\sum_{x \in \gamma_{+}, y \in \gamma_{-}} \phi_{ \pm}(x-y) .
$$

The generator of the birth-and-death dynamics of the stochastic Potts model has the form

$$
\begin{aligned}
& (H F)\left(\gamma_{+}, \gamma_{-}\right) \\
& =\sum_{x \in \gamma_{+}}\left(F\left(\gamma_{+} \backslash x, \gamma_{-}\right)-F\left(\gamma_{+}, \gamma_{-}\right)\right)+\sum_{x \in \gamma_{-}}\left(F\left(\gamma_{+}, \gamma_{-} \backslash x\right)-F\left(\gamma_{+}, \gamma_{-}\right)\right) \\
& \quad+z \int e^{-\beta \sum_{y \in \gamma_{+}} \phi_{+}(x-y)-\beta \sum_{y \in \gamma_{-}} \phi_{ \pm}(x-y)}\left(F\left(\gamma_{+} \cup x, \gamma_{-}\right)-F\left(\gamma_{+}, \gamma_{-}\right)\right) d x \\
& \quad+z \int e^{-\beta \sum_{y \in \gamma_{-}} \phi_{-}(x-y)-\beta \sum_{y \in \gamma_{+}} \phi_{ \pm}(x-y)}\left(F\left(\gamma_{+}, \gamma_{-} \cup x\right)-F\left(\gamma_{+}, \gamma_{-}\right)\right) d x,
\end{aligned}
$$

and as above the existence of the corresponding stochastic process on $\Gamma_{+} \times \Gamma_{-}$follows from the relation between $H$ and associated Dirichlet form, see, e.g., [6]:

$$
\begin{aligned}
\mathcal{E}_{\text {Potts }}(F, F)= & \int_{\Gamma_{+} \times \Gamma_{-}}\left(\sum_{x \in \gamma_{+}}\left|F\left(\gamma_{+} \backslash x, \gamma_{-}\right)-F\left(\gamma_{+}, \gamma_{-}\right)\right|^{2}\right. \\
& \left.+\sum_{x \in \gamma_{-}}\left|F\left(\gamma_{+}, \gamma_{-} \backslash x\right)-F\left(\gamma_{+}, \gamma_{-}\right)\right|^{2}\right) d \mu_{\beta, z}\left(\gamma_{+}, \gamma_{-}\right)
\end{aligned}
$$

The spectral analysis of the generator (2.3) (spectral gap in low density-high temperature regime, construction of invariant subspaces) could be done in the same way as in the paper [4].

Let us note, that the presence of many components in the model permits to consider another action of the dynamics, namely, a change of the component type. However, this action has no a natural description in the stochastic spatial Potts model setting. In the next subsection we introduce a marked continuous system which gives a proper framework for the consideration of mentioned stochastic dynamics.

\subsection{Glauber Dynamics of a Continuous Ising Model}

We will consider here a Glauber type dynamics of continuous marked system, and will study the case when the mark takes only two values. Then the mark has a meaning similar to the spin in classical Ising system and we will name this model a continuous Ising model.

The marked configuration space $\hat{\Gamma}$ of the model is:

$$
\hat{\Gamma}:=\left\{\hat{\gamma}=\left(\gamma, \sigma_{\gamma}\right), \gamma \in \Gamma, \sigma_{\gamma}=\left\{\sigma_{x}(\gamma)\right\}_{x \in \gamma}=\left\{\sigma_{x}\right\}_{x \in \gamma}, \sigma_{x}= \pm 1\right\} .
$$


We consider a reference measure $\mu_{0, z}$ on $\hat{\Gamma}$ with the following decomposition:

$$
d \mu_{0, z}(\hat{\gamma})=d v_{\gamma}\left(\sigma_{\gamma}\right) d \pi_{2 z}(\gamma)
$$

Here we use the Poisson measure $\pi_{2 z}$ on $\gamma$ with activity $2 z$ and the conditional Bernoulli measure (under given configuration $\gamma$ for positions of marks)

$$
d v_{\gamma}\left(\sigma_{\gamma}\right)=\prod_{x \in \gamma} d \nu\left(\sigma_{x}\right)
$$

that is the product of the Bernoulli measures with parameter $p=1 / 2$ over all points from the configuration $\gamma$. Another way to construct this measure is the following one. We consider the extended underlying space $\{-1,+1\} \times \mathbb{R}^{d} \ni \hat{x}=(\sigma, x)$ with the measure $d \hat{x}=d v(\sigma) d x$. Then $\hat{\Gamma} \subset \Gamma\left(\{-1,+1\} \times \mathbb{R}^{d}\right)$ and it is easy to see that the measure $d \mu_{0, z}(\hat{\gamma})$ is nothing but the Poisson measure with the intensity $2 z d \hat{x}$. This measure is defined at first on $\Gamma\left(\{-1,+1\} \times \mathbb{R}^{d}\right)$ and after may be considered on $\hat{\Gamma}$ as on a full measure set.

Let us consider a Gibbs measure on the marked configuration space $\hat{\Gamma}$ (marked Gibbs measure for short). To make our reasoning more clear we assume coupling only between points with different marks, so that the formal Hamiltonian can be written as

$$
U(\hat{\gamma})=\sum_{\hat{x}, \hat{y} \in \hat{\gamma}} \phi(x-y)\left(\sigma_{x}-\sigma_{y}\right)^{2} .
$$

Using the reference measure and this Hamiltonian as above we construct the Gibbs measure $\mu_{\beta, z}$ on $\hat{\Gamma}$.

A generator of a Markov stochastic process involving a spatial birth-and-death process as well as a single-spin flip dynamics on the configurations of spins $\sigma_{\gamma}$ has the following form in the functional space $L^{2}\left(\hat{\Gamma}, \mu_{\beta, z}\right)$

$$
\begin{aligned}
(H F)(\hat{\gamma})= & \sum_{\hat{x} \in \hat{\gamma}}(F(\hat{\gamma} \backslash \hat{x})-F(\hat{\gamma}))+z \int e^{-\beta \sum_{\hat{y} \in \hat{\gamma}} \phi(x-y)\left(\sigma_{x}-\sigma_{y}\right)^{2}}(F(\hat{\gamma} \cup \hat{x})-F(\hat{\gamma})) d \hat{x} \\
& +\lambda \sum_{\hat{x} \in \hat{\gamma}} e^{-\beta \sum_{\hat{y} \in \hat{\gamma}} \phi(x-y)\left(\sigma_{x}+\sigma_{y}\right)^{2}}\left(F\left(\hat{\gamma}^{x}\right)-F(\hat{\gamma})\right),
\end{aligned}
$$

where

$$
\hat{\gamma}^{x}=\left(\gamma, \sigma_{\gamma}^{x}\right)= \begin{cases}\left(y, \sigma_{y}\right), & \text { if } y \neq x, y \in \gamma ; \\ \left(x,-\sigma_{x}\right), & \text { if } y=x, x \in \gamma .\end{cases}
$$

The choice of death and birth rates and spin flip rates depends on a general condition of symmetry for the operator $H$ in the space $L^{2}\left(\hat{\Gamma}, \mu_{\beta, z}\right)$.

The corresponding Dirichlet form can be written as

$$
\begin{aligned}
(-H F, F)= & \mathcal{E}(F, F)=\mathcal{E}_{B A D}(F, F)+\lambda \mathcal{E}_{S F}(F, F) \\
= & \int_{\hat{\Gamma}} \sum_{\hat{x} \in \hat{\gamma}}|F(\hat{\gamma} \backslash \hat{x})-F(\hat{\gamma})|^{2} d \mu_{\beta, z}(\hat{\gamma}) \\
& +\frac{\lambda}{2} \int_{\hat{\Gamma}} \sum_{\hat{x} \in \hat{\gamma}} e^{-\beta \sum_{\hat{y} \in \hat{\gamma}} \phi(x-y)\left(\sigma_{x}+\sigma_{y}\right)^{2}}\left|F\left(\hat{\gamma}^{x}\right)-F(\hat{\gamma})\right|^{2} d \mu_{\beta, z}(\hat{\gamma}) .
\end{aligned}
$$


We denote by $H^{(0)}$ the generator of the birth-and-death part of the dynamics (when $\lambda=0$ in the expression (2.4)):

$$
\left(H^{(0)} F\right)(\hat{\gamma})=\sum_{\hat{x} \in \hat{\gamma}}(F(\hat{\gamma} \backslash \hat{x})-F(\hat{\gamma}))+z \int e^{-\beta \sum_{\hat{y} \in \hat{\gamma}} \phi(x-y)\left(\sigma_{x}-\sigma_{y}\right)^{2}}(F(\hat{\gamma} \cup \hat{x})-F(\hat{\gamma})) d \hat{x}
$$

so that

$$
\left(-H^{(0)} F, F\right)=\mathcal{E}_{B A D}(F, F) .
$$

Let us note that the Gibbs measure is invariant with respect to the space translations on $\hat{\Gamma}$ :

$$
\tau_{s} \hat{\gamma}=\widehat{\gamma+s}=\left\{\widehat{x_{i}+s}, \hat{x}_{i} \in \hat{\gamma}\right\}, \quad x_{i}, s \in \mathbb{R}^{d} .
$$

We denote by $U_{s}$ the corresponding unitary group of the operators of space translations acting in $L^{2}\left(\hat{\Gamma}, \mu_{\beta, z}\right)$ :

$$
\left(U_{s} F\right)(\hat{\gamma})=F\left(\tau_{s}^{-1} \hat{\gamma}\right) .
$$

It easy to see that the operators $U_{s}$ commute with the generator $H(2.4)$.

2.4 Conditions on the Potential $\phi$ and Parameters $\beta, z$

We formulate conditions on the pair potential $\phi$ and parameters $\beta, z$ which guarantee the existence of the Gibbs measure as well as the existence of the first leading invariant subspace of the generator, see Theorem 2 below.

(Ia) (Integrability):

$$
C(\beta):=\int_{\mathbb{R}^{d}}\left|1-e^{-4 \beta \phi(u)}\right| d u<+\infty
$$

(Ib) (Positivity):

$$
\phi(u) \geq 0 \quad \text { for all } u \in \mathbb{R}^{d} .
$$

(Ic) (Low activity-high temperature regime): We assume that the parameter of the model

$$
\varepsilon={ }_{z} C(\beta)<\varepsilon_{0}
$$

is small enough.

\subsection{Main Results}

We state now main results of our paper. Let us denote by $\mathcal{G}_{0}=\{\Psi(\gamma) \equiv c\} \subset L^{2}\left(\hat{\Gamma}, \mu_{\beta, z}\right)$ the subspace of constants. It is easy to see that $\mathcal{G}_{0}$ is an invariant subspace of the operator $H$ and the corresponding eigenvalue is equal to 0 .

Theorem 1 Under assumptions (Ia)-(Ic) and for any $\lambda>0$

$$
(-H F, F) \geq g_{0}(F, F)
$$

for any $F \in L^{2}\left(\hat{\Gamma}, \mu_{\beta, z}\right) \ominus \mathcal{G}_{0}$, where

$$
g_{0}=\left\|\left(\left.H^{(0)}\right|_{L^{2}\left(\hat{\Gamma}, \mu_{\beta, z}\right) \ominus \mathcal{G}_{0}}\right)^{-1}\right\|^{-1} \geq 1-4 \varepsilon .
$$


Theorem 2 Let conditions (Ia)-(Ic) hold, and $\lambda>0$ is small enough. Then the space $L^{2}\left(\hat{\Gamma}, \mu_{\beta, z}\right)$ can be decomposed into a direct orthogonal sum of subspaces invariant with respect to the operator $H$ :

$$
L^{2}\left(\hat{\Gamma}, \mu_{\beta, z}\right)=\mathcal{G}_{0} \oplus \mathcal{G}_{1} \oplus \mathcal{G}_{2}
$$

Let $H_{k}=\left.H\right|_{\mathcal{G}_{k}}, k=0,1,2$, be restrictions of the operator $H$ on the corresponding invariant subspaces $\mathcal{G}_{0}, \mathcal{G}_{1}, \mathcal{G}_{2}$, and $\sigma_{k}=\sigma\left(H_{k}\right)$ be their spectra. Then

$$
\sigma_{0}=\{0\}, \quad \sigma_{1} \subset\left[-1-\gamma_{1},-1+\gamma_{1}\right], \quad \sigma_{2} \subset\left(-\infty,-2+\gamma_{2}\right],
$$

where $\gamma_{1}=3 \varepsilon+4 \lambda, \gamma_{2}=30 \varepsilon+120 \lambda$ are small under small enough $\varepsilon$ and $\lambda$.

Remark The subspace $\mathcal{G}_{1}$ has the following structure:

(1) it is invariant with respect to the generator $H$ and the unitary group of the space translations $\left\{U_{s}, s \in \mathbb{R}^{d}\right\}$ acting in $L^{2}\left(\hat{\Gamma}, d \mu_{\beta, z}\right)$;

(2) the operators $H_{1}=\left.H\right|_{\mathcal{G}_{1}}$ and $U_{s}^{(1)}=\left.U_{s}\right|_{\mathcal{G}_{1}}$ are unitary equivalent to the operators of multiplication by a function (or a matrix function).

Then we call a subspace $\mathcal{G}_{1} \subset L^{2}\left(\hat{\Gamma}, d \mu_{\beta, z}\right)$ one-particle invariant subspace of the generator $H$. In the physical literature the subspace $\mathcal{G}_{1}$ is usually associated with states of "quasi-particles".

Corollary 1 Under small enough $\varepsilon$ and $\lambda$ the spectrum of $H$ is decomposed into at least three isolated parts. As follows from (2.7) at least two gaps exist in the spectrum of $H$. The first spectral gap is a gap between 0 and $\sigma_{1}$, which is estimated by $1-\gamma_{1}$. The latter one is a gap between $\sigma_{1}$ and $\sigma_{2}$.

Corollary 2 Let $F \in L^{2}\left(\hat{\Gamma}, d \mu_{\beta, z}\right) \cap L^{1}\left(\hat{\Gamma}, d \mu_{\beta, z}\right)$ be a function with a non-zero projection on the one-particle invariant subspace $\mathcal{G}_{1}$. Then under conditions of Theorem 2 the correlation function meets the following sandwich estimate as $t \rightarrow \infty$ :

$$
\begin{aligned}
C_{1} e^{-t\left(1+\gamma_{1}\right)} & \leq\langle F(\hat{\gamma}(t)), F(\hat{\gamma}(0))\rangle_{\mathcal{P}} \\
& \equiv\langle F(\hat{\gamma}(t)) \cdot \overline{F(\hat{\gamma}(0))}\rangle_{\mathcal{P}}-\langle F(\hat{\gamma})\rangle_{\mu_{\beta, z}}^{2} \leq C_{2} e^{-t\left(1-\gamma_{1}\right)} .
\end{aligned}
$$

Here $\mathcal{P}$ is the distribution of the process with generator $H$, constant $\gamma_{1}$ is defined in Theorem $2 ; C_{1}, C_{2}$ are constants depending on the function $F$.

In particular, for any function $F_{A}(\hat{\gamma})$ of the form

$$
F_{A}(\hat{\gamma})=\sum_{\hat{x} \in \hat{\gamma}} \chi_{A}(\hat{x}) \sigma_{x},
$$

where $\chi_{A}(\hat{x})=\chi_{A}(x)$ is the characteristic function of a finite volume $A \subset R^{d}$ the following decay of the correlation function holds as $t \rightarrow \infty$ :

$$
\begin{aligned}
C_{1} e^{-t\left(1+\gamma_{1}\right)} & \leq\left\langle F_{A}(\hat{\gamma}(t)), F_{A}(\hat{\gamma}(0))\right\rangle_{\mathcal{P}} \\
& =\left\langle\sum_{\hat{x} \in \hat{\gamma}(t)} \chi_{A}(x) \sigma_{x} \cdot \sum_{\hat{y} \in \hat{\gamma}(0)} \chi_{A}(y) \sigma_{y}\right\rangle_{\mathcal{P}} \leq C_{2} e^{-t\left(1-\gamma_{1}\right)} .
\end{aligned}
$$


Proof Follows the standard reasoning using the spectral theorem, see for example $[3,4]$.

Remark Theorem 1 implies that the estimate from below on the spectral gap is uniform over $\lambda$, and the spectral gap of $H$ is not less then the spectral gap of the generator $H^{(0)}$ for the pure birth-and-death dynamics.

On the other hand, we have to impose an additional assumption on the parameter $\lambda$ to prove the existence of the separated low-lying part of the spectrum $\sigma_{1}$. We don't state the result on $\sigma_{1}$ for any $\lambda>0$ because the approach we used here is based on the perturbation theory for the free generator of the birth-and-death part of the dynamics. We admit that the analogous decomposition of the spectrum could be valid for any $\lambda$, but the analysis of this conjecture requires some modifications of the developed technique.

\subsection{The Space of Quasi-Observables}

Here we formulate main constructions for our model. Let us consider the space of finite configurations

$$
\hat{\Gamma}_{0}:=\bigsqcup_{n=0}^{\infty} \hat{\Gamma}_{0}^{(n)},
$$

where

$$
\hat{\Gamma}_{0}^{(n)}:=\left\{\hat{\eta}=\left(\eta, \sigma_{\eta}\right) \in \hat{\Gamma}_{0}:|\hat{\eta}|=|\eta|=n\right\}
$$

is the space associated with all $n$-point subsets in $\mathbb{R}^{d}$ for $n \in \mathbb{N}$, and $\hat{\Gamma}_{0}^{(0)}:=\{\emptyset\}$. Analogously, we can consider configurations in a finite domain $\Lambda \subset \mathbb{R}^{d}$. For $\hat{\gamma} \in \hat{\Gamma}$ put $\hat{\gamma}_{\Lambda}=\left\{\left(x, \sigma_{x}\right)\right\}_{x \in \gamma_{\Lambda}}$. We will say that $\hat{\gamma}_{1} \subset \hat{\gamma}_{2}$ if $\gamma_{1} \subset \gamma_{2}$ and $\sigma_{x}\left(\gamma_{1}\right)=\sigma_{x}\left(\gamma_{2}\right), x \in \gamma_{1}$.

Denote by $B_{b s}\left(\hat{\Gamma}_{0}\right)$ the space of all complex-valued bounded $\mathcal{B}\left(\hat{\Gamma}_{0}\right)$-measurable functions with bounded support, i.e.,

$$
G \uparrow_{\hat{\Gamma}_{0} \backslash\left(\bigsqcup_{n=0}^{N} \hat{\Gamma}_{\Lambda}^{(n)}\right)} \equiv 0 \text { for some } N \in \mathbb{N} \text {, and some bounded domain } \Lambda \subset \mathbb{R}^{d} .
$$

For any $G \in B_{b s}\left(\hat{\Gamma}_{0}\right)$ we define a function $K G: \hat{\Gamma} \rightarrow \mathbb{C}$ on the space $\hat{\Gamma}$ (so-called $K$ transform) by the following way:

$$
(K G)(\hat{\gamma}):=\sum_{\substack{\hat{\eta} \subset \hat{\gamma} \\|\eta|<\infty}} G(\hat{\eta}) .
$$

Note that for every $G \in B_{b s}\left(\hat{\Gamma}_{0}\right)$ the sum in (2.8) has only a finite number of terms different from zero and thus $K G$ is a well-defined function on $\hat{\Gamma}$. Moreover, if $G \in B_{b s}\left(\hat{\Gamma}_{0}\right)$, then $K G$ is a local function:

$$
(K G)(\hat{\gamma})=(K G)\left(\hat{\gamma}_{\Lambda}\right)
$$

and the function $K G$ is polynomially bounded:

$$
|(K G)(\hat{\gamma})| \leq L\left(1+\left|\hat{\gamma}_{\Lambda}\right|\right)^{N}, \quad \text { for all } \hat{\gamma} \in \hat{\Gamma},
$$


where the bounded domain $\Lambda \subset \mathbb{R}^{d}$ and $N \in \mathbb{N}$ are defined by the function $G$, and $L=$ $\sup _{\xi \in \hat{\Gamma}_{0}}|G(\xi)|$. The inverse mapping of the $K$-transform is defined by

$$
\left(K^{-1} F\right)(\hat{\eta}):=\sum_{\hat{\xi} \subset \hat{\eta}}(-1)^{|\hat{\eta} \backslash \hat{\xi}|} F(\hat{\xi}), \quad \hat{\eta} \in \hat{\Gamma}_{0} .
$$

The functions of the form (2.8) are known as additive type observables or summator functions. Summator functions form a commutative algebra, the product of two summator functions is again a summator function. For every $G_{1}, G_{2} \in B_{b s}\left(\hat{\Gamma}_{0}\right)$ we have

$$
\left(K G_{1}\right) \cdot\left(K G_{2}\right)=K\left(G_{1} \star G_{2}\right)
$$

where the $\star$-convolution is defined on $\mathcal{B}\left(\hat{\Gamma}_{0}\right)$-measurable functions by

$$
\left(G_{1} \star G_{2}\right)(\hat{\eta}):=\sum_{\substack{\left(\hat{\eta}_{1}, \hat{\eta}_{2}, \hat{\eta}_{3}\right): \\ \hat{\eta}_{1} \cup \hat{\eta}_{2} \cup \hat{\eta}_{3}=\hat{\eta}}} G_{1}\left(\hat{\eta}_{1} \cup \hat{\eta}_{2}\right) G_{2}\left(\hat{\eta}_{2} \cup \hat{\eta}_{3}\right), \quad \hat{\eta} \in \hat{\Gamma}_{0},
$$

and $G_{1} \star G_{2} \in B_{b s}\left(\hat{\Gamma}_{0}\right)$, see [5]. Here the summation in (2.10) is over all three mutually disjoint subsets $\left(\hat{\eta}_{1}, \hat{\eta}_{2}, \hat{\eta}_{3}\right)$ of $\hat{\eta}$ which may be empty, such that $\hat{\eta}_{1} \cup \hat{\eta}_{2} \cup \hat{\eta}_{3}=\hat{\eta}$.

\subsection{Correlation Functions}

Let us consider a probability measure $\mu$ defined on $(\hat{\Gamma}, \mathcal{B}(\hat{\Gamma}))$ with finite local moments of all orders. The latter means that for any bounded domain $\Lambda \subset \mathbb{R}^{d}$ holds

$$
\int_{\hat{\Gamma}}\left|\hat{\gamma}_{\Lambda}\right|^{n} d \mu(\hat{\gamma})<\infty \quad \text { for all } n \in \mathbb{N} \text {. }
$$

Then one can define a unique $\sigma$-finite measure $\varrho=\varrho(\mu)$ on $\left(\hat{\Gamma}_{0}, \mathcal{B}\left(\hat{\Gamma}_{0}\right)\right)$, such that

$$
\int_{\hat{\Gamma}}(K G)(\hat{\gamma}) d \mu(\hat{\gamma})=\int_{\hat{\Gamma}_{0}} G(\hat{\eta}) d \varrho(\hat{\eta})
$$

for all $G \in B_{b s}\left(\hat{\Gamma}_{0}\right)$. We call $\varrho$ the correlation measure corresponding to $\mu$. Assume that $\varrho$ is absolute continuous with respect to the Lebesgue-Poisson measure $d \lambda(\hat{\eta})$ on $\hat{\Gamma}_{0}$, where

$$
d \lambda(\hat{\eta})=\frac{1}{n !} d \hat{x}^{\otimes n}
$$

on $\hat{\Gamma}_{0}^{(n)}$. Then there exists the Radon-Nikodym derivative

$$
\varrho_{\mu}(\hat{\eta})=\frac{d \varrho}{d \lambda}(\hat{\eta})
$$

and the functions $\varrho_{\mu}(\hat{\eta}), \hat{\eta} \in \hat{\Gamma}_{0}$ are called the correlation function of the measure $\mu$. In our case of the Gibbs measure under above assumptions on the potential and the parameters of the model, the correlation function exists, and moreover, it meets the following Ruelle bound, see $[11,12]$ :

$$
\varrho_{\mu}(\hat{\eta})<z^{|\eta|}
$$




\subsection{Auxiliary Hilbert Space and Reduced Generator}

Using formulas (2.8-2.9) we have the following representation for the scalar product in $L^{2}\left(\hat{\Gamma}, \mu_{\beta, z}\right)$ of functions $K G_{1}, K G_{2}$ when $G_{1}, G_{2} \in B_{b s}\left(\hat{\Gamma}_{0}\right)$ :

$$
\begin{aligned}
& \left(K G_{1}, K G_{2}\right)_{L^{2}\left(\hat{\Gamma}, \mu_{\beta, z}\right)} \\
& \quad=\int_{\hat{\Gamma}}\left(K G_{1}\right)(\hat{\gamma}) \cdot \overline{\left(K G_{2}\right)(\hat{\gamma})} d \mu_{\beta, z}(\hat{\gamma}) \\
& \quad=\int_{\hat{\Gamma}} K\left(G_{1} \star \overline{G_{2}}\right)(\hat{\gamma}) d \mu_{\beta, z}(\hat{\gamma})=\int_{\hat{\Gamma}_{0}}\left(G_{1} \star \overline{G_{2}}\right)(\hat{\eta}) \varrho_{\mu}(\hat{\eta}) d \lambda(\hat{\eta}) .
\end{aligned}
$$

Since equality (2.12) determines a positive quadratic form in the space $B_{b s}\left(\hat{\Gamma}_{0}\right)$, we can accept the relation

$$
\left(G_{1}, G_{2}\right)=\int_{\hat{\Gamma}_{0}}\left(G_{1} \star \overline{G_{2}}\right)(\hat{\eta}) \varrho_{\mu}(\hat{\eta}) d \lambda(\hat{\eta}), \quad G_{1}, G_{2} \in B_{b s}\left(\hat{\Gamma}_{0}\right)
$$

as a new scalar product. The closure of $B_{b s}\left(\hat{\Gamma}_{0}\right)$ by this scalar product is denoted by $\mathcal{H}$.

It was shown in [5], that the $K$-transform can be extended as a unitary operator

$$
K: \mathcal{H} \rightarrow L^{2}\left(\hat{\Gamma}, \mu_{\beta, z}\right)
$$

Direct calculations give the representation for the unitary image $L:=K^{-1} H K$ of the Glauber generator $H$ acting in the Hilbert space $\mathcal{H}$ :

$$
\begin{aligned}
(L G)(\hat{\eta})= & -|\eta| G(\hat{\eta}) \\
& +z \sum_{\hat{\gamma} \subseteq \hat{\eta}} \int G(\hat{\gamma} \cup \hat{x}) \prod_{\hat{y} \in \hat{\eta} \backslash \hat{\gamma}}\left(e^{-\beta \phi(x-y)\left(\sigma_{x}-\sigma_{y}\right)^{2}}-1\right) \prod_{\hat{y} \in \hat{\gamma}} e^{-\beta \phi(x-y)\left(\sigma_{x}-\sigma_{y}\right)^{2}} d \hat{x} \\
& +\lambda \sum_{\hat{\gamma} \subseteq \hat{\eta}} \sum_{\hat{x} \in \hat{\gamma}}\left(F\left(\hat{\gamma}^{x}\right)-F(\hat{\gamma})\right) \prod_{\hat{y} \in \hat{\gamma} \backslash \hat{x}} e^{-\beta \phi(x-y)\left(\sigma_{x}+\sigma_{y}\right)^{2}} \\
& \times \prod_{\hat{y} \in \hat{\eta} \backslash \hat{\gamma}}\left(e^{-\beta \phi(x-y)\left(\sigma_{x}+\sigma_{y}\right)^{2}}-1\right) .
\end{aligned}
$$

We call the operator (2.14) the reduced generator, and in what follows we will study the spectral properties of the operator $L$ in the space $\mathcal{H}$. We denote by $L^{(0)}$ the operator $L$ for $\lambda=0$ (the generator of the pure birth-and-death part of the dynamics):

$$
\begin{aligned}
\left(L^{(0)} G\right)(\hat{\eta})= & \left(K^{-1} H^{(0)} K G\right)(\hat{\eta}) \\
= & -|\eta| G(\hat{\eta})+z \sum_{\hat{\gamma} \subseteq \hat{\eta}} \int G(\hat{\gamma} \cup \hat{x}) \prod_{\hat{y} \in \hat{\eta} \backslash \hat{\gamma}}\left(e^{-\beta \phi(x-y)\left(\sigma_{x}-\sigma_{y}\right)^{2}}-1\right) \\
& \times \prod_{\hat{y} \in \hat{\gamma}} e^{-\beta \phi(x-y)\left(\sigma_{x}-\sigma_{y}\right)^{2}} d \hat{x} .
\end{aligned}
$$




\subsection{Main Results in Terms of the Reduced Generator $L$}

We formulate here the main results in terms of the auxiliary Hilbert space $\mathcal{H}$ and the operator $L$. As follows from the unitary property (2.13) of the $K$-transform, statements of Theorems 3 and 4 below are equivalent to Theorems 1 and 2 .

Let $\mathcal{H}_{0} \subset \mathcal{H}$ be an one-dimensional subspace, generated by the "vacuum" vector $\Phi_{0}$ :

$$
\Phi_{0}(\hat{\eta})= \begin{cases}1, & \hat{\eta}=\varnothing \\ 0, & \hat{\eta} \neq \varnothing .\end{cases}
$$

It is easy to see, that $L \Phi_{0}=0$.

Theorem 3 Under assumptions (Ia)-(Ic) and for any $\lambda>0$

$$
(-L G, G) \geq g_{0}(G, G), \quad G \in \mathcal{H}_{0}^{\perp}=\mathcal{H} \ominus \mathcal{H}_{0},
$$

where $g_{0}$ is the spectral gap of the operator $L^{(0)}$ :

$$
g_{0}=\left\|\left(\left.L^{(0)}\right|_{\mathcal{H} \ominus \mathcal{H}_{0}}\right)^{-1}\right\|^{-1} \geq 1-4 \varepsilon .
$$

Theorem 4 Let assumptions (Ia)-(Ic) be valid and $\lambda$ is small enough. Then the space $\mathcal{H}$ can be decomposed into a direct orthogonal sum

$$
\mathcal{H}=\hat{\mathcal{H}}_{0} \oplus \hat{\mathcal{H}}_{1} \oplus \hat{\mathcal{H}}_{2}
$$

of the subspaces $\hat{\mathcal{H}}_{0}=\mathcal{H}_{0}, \hat{\mathcal{H}}_{1}, \hat{\mathcal{H}}_{2}$ invariant with respect to the operator $L$. Let $L_{k}=\left.L\right|_{\hat{\mathcal{H}}_{k}}$, $k=0,1,2$, be restrictions of the operator $L$ on the corresponding subspaces $\hat{\mathcal{H}}_{0}, \hat{\mathcal{H}}_{1}, \hat{\mathcal{H}}_{2}$, and $\sigma_{k}=\sigma\left(L_{k}\right)$ be their spectra. Then

$$
\sigma_{0}=\{0\}, \quad \sigma_{1} \subset\left[-1-\gamma_{1},-1+\gamma_{1}\right], \quad \sigma_{2} \subset\left(-\infty,-2+\gamma_{2}\right],
$$

where $\gamma_{1}=3 \varepsilon+4 \lambda, \gamma_{2}=30 \varepsilon+120 \lambda$ are small under small enough $\varepsilon$ and $\lambda$.

\section{Proof of Theorems 3, 4. A General Scheme of the Spectral Analysis of the Generator}

We denote by $C_{b s}\left(\hat{\Gamma}_{0}\right)$ the set of all continuous functions on $\hat{\Gamma}_{0}$ with bounded support, and let us consider the following norm in the space $C_{b s}\left(\hat{\Gamma}_{0}\right)$ :

$$
\|G\|_{M}=\sup _{\hat{\eta}}\left(\left(\frac{1}{3}\right)^{|\eta|} \int_{\Gamma_{0}}(|\eta|+|\xi|) \sup _{\sigma_{\xi}}|G(\hat{\eta} \cup \hat{\xi})| M^{|\xi|} d \xi\right)+|G(\emptyset)|
$$

where $G \in C_{b s}\left(\hat{\Gamma}_{0}\right)$ and $d \xi$ is the Lebesgue-Poisson measure on the space of finite configurations $\Gamma_{0}$. We take a constant $M$, such that $M>4 z$.

We denote by $\mathcal{L}$ a closure of $C_{b s}\left(\hat{\Gamma}_{0}\right)$ with respect to the norm (3.1). Let us note that the Banach space $\mathcal{L}$ and the norm (3.1) are invariant with respect to the operators $U_{t}$ of the space translations:

$$
U_{t} G \in \mathcal{L}, \quad\left\|U_{t} G\right\|_{M}=\|G\|_{M}
$$

for any $G \in \mathcal{L}$ and any $t \in \mathbb{R}^{v}$. 


\section{Lemma 3.1 Let}

$$
M>4 z,
$$

then $\mathcal{L} \subset \mathcal{H}$, the space $\mathcal{L}$ is dense in $\mathcal{H}$, and

$$
\|G\|_{\mathcal{H}} \leq\|G\|_{M}, \quad G \in \mathcal{L}
$$

Proof of Lemma 3.1: see Sect. 4.

We denote the domain of the operator $L$ in $\mathcal{H}$ by $D_{L} \subset \mathcal{H}$. Let us consider the following set of functions

$$
\widetilde{D_{L}}=\left\{G \in \mathcal{L} \cap D_{L}: L G \in \mathcal{L} .\right\}
$$

Then $\widetilde{D_{L}}$ is the domain of $L$ as an operator acting in $\mathcal{L}$. Since $C_{b s}\left(\Gamma_{0}\right) \subset D_{L}$ and $C_{b s}\left(\Gamma_{0}\right) \subset$ $\widetilde{D_{L}}$, then $\widetilde{D_{L}}$ is dense in $\mathcal{L}$.

For any $k=0,1,2, \ldots$, we define the following spaces of functions:

$$
\begin{gathered}
\mathcal{L}_{k}=\{G \in \mathcal{L}: G(\hat{\eta})=0, \text { when }|\eta| \neq k\}, \\
\mathcal{L}_{\geq k}=\bigoplus_{j \geq k} \mathcal{L}_{j}=\{G \in \mathcal{L}: G(\hat{\eta})=0,|\eta|<k\}, \\
\mathcal{L}_{\leq k}=\bigoplus_{j \leq k} \mathcal{L}_{j}=\{G \in \mathcal{L}: G(\hat{\eta})=0,|\eta|>k\} .
\end{gathered}
$$

All these subspaces are closed in $\mathcal{L}$. By analogy we can define subspaces $\mathcal{H}_{k}, \mathcal{H}_{\geq k}, \mathcal{H}_{\leq k} \subset$ $\mathcal{H}$, which are also closed in the space $\mathcal{H}$.

We describe now a general scheme of the spectral analysis of the generator. Let us consider a decomposition of $\mathcal{L}$ in a direct sum of two subspaces

$$
\mathcal{L}=\mathcal{R}_{1} \oplus \mathcal{R}_{2}
$$

This decomposition implies the following matrix representation for the operator $L$ :

$$
L=\left(\begin{array}{ll}
L_{11} & L_{12} \\
L_{21} & L_{22}
\end{array}\right)
$$

where $L_{11}: \mathcal{R}_{1} \rightarrow \mathcal{R}_{1}, L_{12}: \mathcal{R}_{2} \rightarrow \mathcal{R}_{1}$ etc.

We will construct an invariant to the operators $L$ subspace $\hat{\mathcal{R}}_{1}$ as the graph of a bounded operator $S: \mathcal{R}_{1} \rightarrow \mathcal{R}_{2}$ :

$$
\hat{\mathcal{R}}_{1}=\left\{G+S G ; G \in \mathcal{R}_{1}\right\}, \quad S G \in \mathcal{R}_{2},
$$

(see the general description of this approach in $[3,7,8]$ ). The condition of the invariance of the subspace $\hat{\mathcal{R}}_{1}$ with respect to $L$ could be rewritten as the following equation on the operator $S$ :

$$
S=-L_{22}^{-1} L_{21}+L_{22}^{-1} S L_{11}+L_{22}^{-1} S L_{12} S .
$$

The next step of the scheme is to write the representation for $L$ restricted to the invariant subspace $\hat{\mathcal{R}}_{1}$. We consider the projection operator

$$
P_{1}: \hat{\mathcal{R}}_{1} \rightarrow \mathcal{R}_{1}, \quad P_{1}(G+S G)=G \in \mathcal{R}_{1},
$$


and the inverse operator

$$
P_{1}^{-1}: \mathcal{R}_{1} \rightarrow \hat{\mathcal{R}}_{1}, \quad P_{1}^{-1} G=G+S G
$$

According to the construction of the invariant subspace (3.7) the operator $\left.L\right|_{\hat{\mathcal{R}}_{1}}$ can be written as

$$
\left.L\right|_{\hat{\mathcal{R}}_{1}}=P_{1}^{-1}\left(L_{11}+L_{12} S\right) P_{1},
$$

and analogously, for the inverse operator $\left(\left.L\right|_{\hat{\mathcal{R}}_{1}}\right)^{-1}$ we have

$$
\left(\left.L\right|_{\hat{\mathcal{R}}_{1}}\right)^{-1}=P_{1}^{-1}\left(L_{11}+L_{12} S\right)^{-1} P_{1} .
$$

It is clear from (3.9-3.10) that the norm of the operator $\left.L\right|_{\hat{\mathcal{R}}_{1}}$ can be estimated in terms of the norms of the operators $S, P_{1}, P_{1}^{-1}, L_{11}, L_{12}$. In many situations it will be more easy done in the norm of the Banach space $\mathcal{L}$.

The last step is to obtain estimates in the Hilbert space $\mathcal{H}$. Here we used the following result.

Proposition Let $\mathcal{L}$ be a Banach space with a norm $\|\cdot\|_{\mathcal{L}}$, such that $\mathcal{L} \subset \mathcal{H}$ is a dense subset of a Hilbert space $\mathcal{H}$, and for any $f \in \mathcal{L}$

$$
\|f\|_{\mathcal{H}} \leq\|f\|_{\mathcal{L}}
$$

Let $L$ be a self-adjoint operator in $\mathcal{H}$ such that $L \mathcal{L} \subset \mathcal{L}$ and the restriction $\left.L\right|_{\mathcal{L}}$ is a bounded operator in $\mathcal{L}$. Then $L$ is a bounded operator in $\mathcal{H}$, and

$$
\|L\|_{\mathcal{H}} \leq\|L\|_{\mathcal{L}}
$$

Proof See $[3,8]$.

We will apply now this scheme to the proofs of Theorems 3-4. To find the bound on the spectral gap in Theorem 3 we will estimate the norm of the inverse operator $L^{-1}$ on the subspace orthogonal to the constant subspace. To do this we consider the decomposition of $\mathcal{L}$ in a direct sum

$$
\mathcal{L}=\mathcal{L}_{0} \oplus \mathcal{L}_{\geq 1}
$$

then the operator $L^{(0)}$ has a matrix representation:

$$
L^{(0)}=\left(\begin{array}{ll}
0 & L_{01} \\
0 & L_{11}
\end{array}\right)
$$

with $L_{01}: \mathcal{L}_{\geq 1} \rightarrow \mathcal{L}_{0}, L_{11}: \mathcal{L}_{\geq 1} \rightarrow \mathcal{L}_{\geq 1}$. The subspace $\mathcal{L}_{0}$ is invariant for $L^{(0)}$, and we construct an invariant subspace $\hat{\mathcal{L}}_{\geq 1}$ complementary to $\mathcal{L}_{0}$ as a graph of an operator $T: \mathcal{L}_{\geq 1} \rightarrow \mathcal{L}_{0}:$

$$
\hat{\mathcal{L}}_{\geq 1}=\mathcal{L}_{\geq 1}+T \mathcal{L}_{\geq 1}
$$

The condition of the invariance implies that

$$
T=L_{01} L_{11}^{-1} .
$$


Lemma 3.2 For all small enough $\varepsilon$

$$
\left\|L_{01}\right\|_{M} \leq \varepsilon
$$

where $\|\mid \cdot\|_{M}$ means the operator norm, generated by the norm $\|\cdot\|_{M}$ in the Banach space $\mathcal{L}$.

Proof See Sect. 4.

Lemma 3.3 For all small enough $\varepsilon$

$$
\left\|L_{11}^{-1} \mid\right\|_{M} \leq \frac{1}{1-e \varepsilon} .
$$

Proof See Sect. 4.

Thus, Lemmas 3.2-3.3 and representations (3.14-3.15) imply the following estimates

$$
\|T\|_{M} \leq \frac{\varepsilon}{1-e \varepsilon}, \quad\left\|P_{\geq 1}^{-1} \mid\right\|_{M} \leq 1+\frac{\varepsilon}{1-e \varepsilon}, \quad\left\|P_{\geq 1}\right\|_{M} \leq 1,
$$

with

$$
P_{\geq 1}: \hat{\mathcal{L}}_{\geq 1} \rightarrow \mathcal{L}_{\geq 1}, \quad P_{\geq 1}^{-1}: \mathcal{L}_{\geq 1} \rightarrow \hat{\mathcal{L}}_{\geq 1},
$$

and using these estimates together with (3.10) and (3.13) we have

$$
\left\|\left(\left.L^{(0)}\right|_{\hat{\mathcal{L}}_{\geq 1}}\right)^{-1} \mid\right\|_{M} \leq \frac{1-(e-1) \varepsilon}{(1-e \varepsilon)^{2}} .
$$

Then from (3.11) and (3.17) it follows that for any $G \in \mathcal{H} \ominus \mathcal{H}_{0}$

$$
\left(-L^{(0)} G, G\right) \geq g_{0}(G, G)
$$

where

$$
g_{0}=\left\|\left(\left.L^{(0)}\right|_{\mathcal{H} \ominus \mathcal{H}_{0}}\right)^{-1}\right\|_{\mathcal{H}}^{-1} \geq\|\|\left(\left.L^{(0)}\right|_{\hat{\mathcal{L}}_{\geq 1}}\right)^{-1} \mid \|_{M}^{-1} \geq 1-4 \varepsilon,
$$

and the last bound is valid under small enough $\varepsilon$. Finally, using that the operator (2.4) is associated with the sum (2.5) of two Dirichlet forms $\mathcal{E}_{B A D}(F, F)$ and $\mathcal{E}_{S F}(F, F)$, we have for any $F \in L^{2}\left(\hat{\Gamma}, \mu_{\beta, z}\right)$ with $<F>_{\mu_{\beta, z}}=0$

$$
\begin{aligned}
(-L G, G) & =(-H F, F)_{L^{2}}=\mathcal{E}_{B A D}(F, F)+\lambda \mathcal{E}_{S F}(F, F) \geq \mathcal{E}_{B A D}(F, F) \\
& =\left(-H^{(0)} F, F\right)_{L^{2}}=\left(-L^{(0)} G, G\right) \geq g_{0}(G, G) .
\end{aligned}
$$

Theorem 3 is proved completely.

To separate a low-lying part of the spectrum of the operator $L$ (the so-called one-particle branch of the spectrum) we should consider another decomposition of $\mathcal{L}$ into a direct sum

$$
\mathcal{L}=\mathcal{L}_{\leq 1} \oplus \mathcal{L}_{\geq 2}
$$

That implies the following matrix representation for the operator $L$ :

$$
L=\left(\begin{array}{ll}
L_{11} & L_{12} \\
L_{21} & L_{22}
\end{array}\right)
$$

where $L_{11}: \mathcal{L}_{\leq 1} \rightarrow \mathcal{L}_{\leq 1}, L_{12}: \mathcal{L}_{\geq 2} \rightarrow \mathcal{L}_{\leq 1}$ etc. 
Following the above scheme we construct the invariant subspace

$$
\hat{\mathcal{L}}_{\leq 1}=\left\{G+S G, G \in \mathcal{L}_{\leq 1}\right\}
$$

as the graph (3.7) of a bounded operator $S: \mathcal{L}_{\leq 1} \rightarrow \mathcal{L}_{\geq 2}$ that is a solution of the equation (3.8). To prove the existence of $S$ with a small norm we have to estimate the norms of the operators from equation (3.8).

Lemma 3.4 For all small enough $\varepsilon$ and $\lambda$ the operator $L_{22}$ is reversible in $\mathcal{L}_{\geq 2}$, and the norm of the operator $L_{22}^{-1}$ has the upper bound

$$
\left\|\mid L_{22}^{-1}\right\|_{M}<\frac{1}{2}(1+3 \varepsilon+6 \lambda) .
$$

Proof See Sect. 4.

Lemma 3.5 For small enough $\varepsilon$ and $\lambda$ we have

$$
\begin{gathered}
\left\|\mid L_{11}\right\| \|_{M}<1+2 \varepsilon+2 \lambda, \\
\left\|\mid L_{12}\right\|_{M}<\varepsilon, \\
\left\|\mid L_{21}\right\|_{M}<4 \varepsilon+36 \lambda .
\end{gathered}
$$

Proof See Sect. 4.

We denote by $\mathcal{F}(S)$ the right-hand side of (3.8) and consider the mapping $S \rightarrow \mathcal{F}(S)$ in the space of bounded linear operators $\mathcal{O}_{1,2}$, acting from $\mathcal{L}_{\leq 1}$ to $\mathcal{L}_{\geq 2}$. Let $\mathcal{B}_{\delta} \subset \mathcal{O}_{1,2}$ be a ball in the space $\mathcal{O}_{1,2}$ of the radius $\delta$ :

$$
\mathcal{B}_{\delta}=\left\{S \in \mathcal{O}_{1,2}:\left|\|S \mid\|_{M}<\delta\right\}\right.
$$

Then estimates (3.21-3.24) imply the following result.

Lemma 3.6 Under small enough $\varepsilon$ and $\lambda$ the ball $\mathcal{B}_{\delta}$ with $\delta=8 \varepsilon+48 \lambda$ is invariant with respect to $\mathcal{F}$ :

$$
\mathcal{F} \mathcal{B}_{\delta} \subseteq \mathcal{B}_{\delta},
$$

and the mapping $\mathcal{F}(S)$ is a contraction on $\mathcal{B}_{\delta}$ :

$$
\|\| \mathcal{F}\left(S_{1}\right)-\mathcal{F}\left(S_{2}\right)\|\|_{M} \leq c\left\|\mid S_{1}-S_{2}\right\| \|_{M}, \quad S_{1}, S_{2} \in \mathcal{B}_{\delta},
$$

with $0<c<1$.

\section{Proof See Sect. 4.}

Lemma 3.6 implies the existence and the uniqueness of the solution $S$ of the equation (3.8) with a small norm

$$
\|||\|_{M}<\delta=8 \varepsilon+48 \lambda .
$$


Therefore, we constructed the subspace $\hat{\mathcal{L}}_{\leq 1}$ of the form (3.20), which is invariant with respect to the operator $L$. We denote by $L_{\leq 1}=\left.L\right|_{\hat{\mathcal{L}}_{\leq 1}}$ the restriction of $L$ to this invariant subspace.

The second "supplementary" invariant subspace $\hat{\mathcal{L}}_{\geq 2}$ of the form

$$
\hat{\mathcal{L}}_{\geq 2}=\left\{G+T G ; G \in \mathcal{L}_{\geq 2}\right\}, \quad T: \mathcal{L}_{\geq 2} \rightarrow \mathcal{L}_{\leq 1}
$$

can be constructed using the same reasoning as above, see also constructions from [4], and in addition the norm of the operator $T$ can be estimated as

$$
\|\mid\| T \|_{M}<8 \varepsilon+48 \lambda .
$$

Lemma 3.7 The following decomposition into a direct sum of invariant subspaces holds for any small enough $\varepsilon$ and $\lambda$ :

$$
\mathcal{L}=\hat{\mathcal{L}}_{\leq 1}+\hat{\mathcal{L}}_{\geq 2}
$$

Proof See Sect. 4.

We denote by $L_{2}=\left.L\right|_{\hat{\mathcal{L}}_{\geq 2}}$.

Lemma 3.8 Let $\varepsilon$ and $\lambda$ be small enough, then the operator $L_{2}$ is reversible in $\hat{\mathcal{L}}_{\geq 2}$ and

$$
\left\|L_{2}^{-1} \mid\right\|_{M} \leq \frac{1}{2}(1+14 \varepsilon+58 \lambda) .
$$

Proof See Sect. 4.

Estimate (3.31) implies inclusion (2.18) for the location of the spectrum $\sigma_{2}$. The next step of the proof of Theorem 4 is to find the location of the first isolated part of the spectrum. As follows from our constructions, the space $\hat{\mathcal{L}}_{\leq 1}$ contains the one-dimensional invariant subspace of constants $\mathcal{L}_{0}=\left\{\Phi_{0}\right\}$, such that $L \mathcal{L}_{0}=0$. We denote by $\hat{\mathcal{L}}_{1}$ the following subspace of $\hat{\mathcal{L}}_{\leq 1}$ :

$$
\hat{\mathcal{L}}_{1}=\mathcal{H}_{0}^{\perp} \cap \hat{\mathcal{L}}_{\leq 1},
$$

where $\mathcal{H}_{0}^{\perp}$ is the orthogonal complement in $\mathcal{H}$ to $\mathcal{H}_{0}$, and $\hat{\mathcal{L}}_{1}$ is invariant with respect to the operator $L$ as an intersection of two invariant subspaces. Then $\sigma_{1}$ is defined as a spectrum of the operator $\left.L\right|_{\hat{\mathcal{L}}_{1}}$ restricted to the invariant subspace $\hat{\mathcal{L}}_{1}$.

The representations (3.20) and (3.32) implies that the subspace $\hat{\mathcal{L}}_{1}$ can be determined again as a graph

$$
\hat{\mathcal{L}}_{1}=\left\{G_{1}+S^{\prime} G_{1} ; G_{1} \in \mathcal{L}_{1}\right\}
$$

of an operator $S^{\prime}: \mathcal{L}_{1} \rightarrow \mathcal{L}_{\geq 2} \oplus \mathcal{L}_{0}$, where

$$
S^{\prime} G_{1}=\left.S\right|_{\mathcal{L}_{1}} G_{1}+C_{0}\left(G_{1}\right) \Phi_{0} \in \mathcal{L}_{\geq 2} \oplus \mathcal{L}_{0}, \quad G_{1} \in \mathcal{L}_{1}, \quad S: \mathcal{L}_{\leq 1} \rightarrow \mathcal{L}_{\geq 2},
$$

and $C_{0}\left(G_{1}\right)$ is a projection of $G_{1}+\left.S\right|_{\mathcal{L}_{1}} G_{1}$ to the space $\mathcal{H}_{0}$ :

$$
C_{0}\left(G_{1}\right)=-\left(G_{1}+\left.S\right|_{\mathcal{L}_{1}} G_{1}, \Phi_{0}\right)_{\mathcal{H}}=-\varrho_{1} \int G_{1}(\hat{x}) d \hat{x}-\int_{|\eta| \geq 2}\left(\left.S\right|_{\mathcal{L}_{1}} G_{1}\right)(\hat{\eta}) \varrho(\hat{\eta}) d \hat{\eta} .
$$


That implies the following upper bound:

$$
\left|C_{0}\left(G_{1}\right)\right|<\left(\varepsilon+\||| S \mid\|_{M}\right)\left\|G_{1}\right\|_{M},
$$

and (3.27), (3.34) come to the estimate

$$
\left\|S^{\prime}\right\|_{M} \leq 17 \varepsilon+96 \lambda .
$$

Thus we established the decomposition:

$$
\mathcal{L}=\mathcal{L}_{0}+\hat{\mathcal{L}}_{1}+\hat{\mathcal{L}}_{\geq 2}
$$

Using the same reasoning as above we obtain the following representation for the operator $L_{1}$ :

$$
L_{1}=\left.L\right|_{\hat{\mathcal{L}}_{1}}=P_{1}^{-1}\left(L_{11}^{\prime}+L_{12}\left(\left.S\right|_{\mathcal{L}_{1}}\right)\right) P_{1},
$$

where $L_{11}^{\prime}: \mathcal{L}_{1} \rightarrow \mathcal{L}_{1}$

$$
P_{1}: \hat{\mathcal{L}}_{1} \rightarrow \mathcal{L}_{1}, \quad P_{1} G=G_{1} \in \mathcal{L}_{1},
$$

and the inverse operator

$$
P_{1}^{-1}: \mathcal{L}_{1} \rightarrow \hat{\mathcal{L}}_{1}, \quad P_{1}^{-1} G_{1}=G_{1}+S G_{1}+C_{0}\left(G_{1}\right) \Phi_{0}=G_{1}+S^{\prime} G_{1}
$$

Then using (3.35) we can estimate the norms of the operators $P_{1}, P_{1}^{-1}$ and $L_{1}$ in the space $\mathcal{L}$, and eventually to find the location of the spectra from Theorem 4 , for details, see the proof of Lemma 3.9. We introduce the subspaces $\hat{\mathcal{H}}_{1}, \hat{\mathcal{H}}_{\geq 2}$ as the closure in $\mathcal{H}$ of the subspaces $\hat{\mathcal{L}}_{1}, \hat{\mathcal{L}}_{\geq 2}$ respectively.

Lemma 3.9 The subspaces $\hat{\mathcal{H}}_{1}$ and $\hat{\mathcal{H}}_{\geq 2}$ are invariant with respect to the operator L. Together with the invariant subspace $\mathcal{H}_{0}$ they give the orthogonal decomposition (2.17) of the space $\mathcal{H}$. In addition, the spectra of $L$ on the corresponding subspaces meet the condition (2.18).

\section{Proof See Sect. 4.}

\section{Proofs of Lemmas}

\subsection{Proof of Lemma 3.1}

To prove estimate (3.4) we follow the similar reasoning as in our paper [4]. We prove (3.4) first for the functions $G \in \mathcal{L}$, such that $G(\emptyset)=0$. Using the estimate on the correlation function $\rho\left(\hat{\eta}_{1} \cup \hat{\eta}_{2} \cup \hat{\eta}_{3}\right)<z^{\left|\eta_{1}\right|+\left|\eta_{2}\right|+\left|\eta_{3}\right|}$, we have:

$$
\begin{aligned}
\|G\|_{\mathcal{H}}^{2} & =\iiint G\left(\hat{\eta}_{1} \cup \hat{\eta}_{2}\right) G\left(\hat{\eta}_{2} \cup \hat{\eta}_{3}\right) \rho\left(\hat{\eta}_{1} \cup \hat{\eta}_{2} \cup \hat{\eta}_{3}\right) d \hat{\eta}_{1} d \hat{\eta}_{2} d \hat{\eta}_{3} \\
& \leq \iiint\left|G\left(\hat{\eta}_{2} \cup \hat{\eta}_{3}\right)\right| z^{\left|\eta_{3}\right|}\left(\frac{1}{3}\right)^{\left|\eta_{2}\right|}\left|G\left(\hat{\eta}_{1} \cup \hat{\eta}_{2}\right)\right|(3 z)^{\left|\eta_{2}\right|} z^{\left|\eta_{1}\right|} d \hat{\eta}_{1} d \hat{\eta}_{2} d \hat{\eta}_{3}
\end{aligned}
$$




$$
\begin{aligned}
& \leq \sup _{\hat{\eta}_{2}}\left(\left(\frac{1}{3}\right)^{\left|\eta_{2}\right|} \int \sup _{\sigma_{\eta_{3}}}\left|G\left(\hat{\eta}_{2} \cup \hat{\eta}_{3}\right)\right|\left(\left|\eta_{2}\right|+\left|\eta_{3}\right|\right)\left(\frac{z}{M}\right)^{\left|\eta_{3}\right|} M^{\left|\eta_{3}\right|} d \eta_{3}\right) \\
& \times \iint \sup _{\sigma_{\eta_{1}} \cup \eta_{2}}\left|G\left(\hat{\eta}_{1} \cup \hat{\eta}_{2}\right)\right|\left(\frac{3 z}{M}\right)^{\left|\eta_{2}\right|}\left(\frac{z}{M}\right)^{\left|\eta_{1}\right|} M^{\left|\eta_{1}\right|+\left|\eta_{2}\right|} d \eta_{1} d \eta_{2} \\
& \leq\|G\|_{M} \cdot \int \sup _{\sigma_{\varepsilon}}|G(\hat{\varepsilon})|\left(\sum_{\substack{\eta_{1} \subseteq \varepsilon \\
\varepsilon=\eta_{1} \cup \eta_{2}}}\left(\frac{3 z}{M}\right)^{\left|\eta_{2}\right|}\left(\frac{z}{M}\right)^{\left|\eta_{1}\right|}\right) M^{|\varepsilon|} d \varepsilon .
\end{aligned}
$$

In the last inequality we applied the well-known formula, see [10]

$$
\int F\left(\xi_{1} \cup \xi_{2}\right) \varphi_{1}\left(\xi_{1}\right) \varphi_{2}\left(\xi_{2}\right) d \xi_{1} d \xi_{2}=\int F(\xi) \sum_{\xi_{1} \subseteq \xi} \varphi_{1}\left(\xi_{1}\right) \varphi_{2}\left(\xi \backslash \xi_{1}\right) d \xi .
$$

Using the equality

$$
\sum_{\substack{\eta_{1} \cup \eta_{2}=\varepsilon \\ \eta_{1} \cap \eta_{2}=\emptyset}}\left(\frac{3 z}{M}\right)^{\left|\eta_{1}\right|}\left(\frac{z}{M}\right)^{\left|\eta_{2}\right|}=\left(\frac{3 z}{M}+\frac{z}{M}\right)^{|\varepsilon|}=\left(\frac{4 z}{M}\right)^{|\varepsilon|},
$$

and the condition $M \geq 4 z$ (together with the apparent inequality $\frac{z}{M} \leq\left(\frac{1}{3}\right)$ ) we have, that the expression (4.1) can be estimated from above by

$$
\|G\|_{M} \cdot \int \sup _{\sigma_{\varepsilon}}|G(\hat{\varepsilon})| M^{|\varepsilon|} d \varepsilon \leq\|G\|_{M}^{2} .
$$

The estimate in the general case, when the function $G \in \mathcal{L}$ can be represented as a sum $G=g \Phi_{0}+G_{1}$ of the "vacuum" vector $\Phi_{0}$ and a function $G_{1}$ such that $G_{1}(\emptyset)=0$, easily follows from the above reasoning and the Cauchy-Schwarz-Bunyakovskii inequality. Thus, estimate (3.4) holds together with inclusion $\mathcal{L} \subset \mathcal{H}$. Since the space $C_{b s}\left(\hat{\Gamma}_{0}\right)$ of continuous functions on $\hat{\Gamma}_{0}$ with bounded support is contained in $\mathcal{L}$, and $C_{b s}$ is dense in $\mathcal{H}$, then $\mathcal{L}$ is dense in $\mathcal{H}$. Lemma is completely proved.

\subsection{Proof of Lemma 3.2}

Representations (2.15), (3.13) and (3.1) imply that

$$
\left(L_{01} G\right)_{0}=z \int G_{1}(\hat{x}) d \hat{x}
$$

and

$$
\left\|G_{1}\right\|_{M}=\sup \left\{\frac{1}{3} \sup _{\hat{x}}\left|G_{1}(\hat{x})\right| ; \int \sup _{\sigma_{x}}\left|G_{1}(\hat{x})\right| M d x\right\} .
$$

Consequently, taking $M=\frac{1}{C(\beta)}$ we have

$$
\left\|L_{01}\right\|_{M} \leq \frac{z}{M}=\varepsilon .
$$




\subsection{Proof of Lemma 3.3}

We refer for the proof to the next section (proof of Lemma 3.4) where the general case is under consideration. The upper bound on the norm of the operator $L_{11}^{-1}$ could be found using the same reasoning as in Lemma 3.4 for bound (3.21) on the norm of $L_{22}^{-1}$. The operator $L_{11}^{-1}$ can be written in the notations of Lemma 3.4. as

$$
L_{11}^{-1}=\left(L_{11}^{0}+L_{11}^{1}\right)^{-1}=\left(E_{\geq 1}+\left(L_{11}^{0}\right)^{-1} L_{11}^{1}\right)^{-1}\left(L_{11}^{0}\right)^{-1},
$$

and (3.16) immediately follows from the estimate on $\left\|\mid\left(L_{11}^{0}\right)^{-1} L_{11}^{1}\right\| \|_{M}$, which is the same as (4.14).

\subsection{Proof of Lemma 3.4}

We consider the following decomposition for the operator $L$ in the sum of operators:

$$
L=L^{0}+L^{1}+L^{2}
$$

where

$$
\left(L^{0} G\right)(\hat{\eta})=-|\eta| G(\hat{\eta})
$$

is a "free" generator, and the "perturbations" $L^{1}$ and $L^{2}$ are given as

$$
\left(L^{1} G\right)(\hat{\eta})=z \sum_{\hat{\gamma} \subseteq \hat{\eta}} \int G(\hat{\gamma} \cup \hat{x}) \prod_{\hat{y} \in \hat{\eta} \backslash \hat{\gamma}}\left(e^{-\beta \varphi(x-y)\left(\sigma_{x}-\sigma_{y}\right)^{2}}-1\right) \prod_{\hat{y}^{\prime} \in \hat{\gamma}} e^{-\beta \varphi\left(x-y^{\prime}\right)\left(\sigma_{x}-\sigma_{y^{\prime}}\right)^{2}} d \hat{x},
$$

and

$$
\begin{aligned}
\left(L^{2} G\right)(\hat{\eta})= & \lambda \sum_{\hat{\gamma} \subseteq \hat{\eta}} \sum_{\hat{x} \in \hat{\gamma}}\left(G\left(\hat{\gamma}^{x}\right)-G(\hat{\gamma})\right) \prod_{\hat{y} \in \hat{\gamma} \backslash \hat{x}} e^{-\beta \varphi(x-y)\left(\sigma_{x}+\sigma_{y}\right)^{2}} \\
& \times \prod_{\hat{y} \in \hat{\eta} \hat{\gamma}}\left(e^{-\beta \varphi(x-y)\left(\sigma_{x}+\sigma_{y}\right)^{2}}-1\right) .
\end{aligned}
$$

Here we assume that $\prod_{\hat{y} \in \emptyset} f(y)=1$. By analogy with the matrix representation (3.19) associated with the decomposition (3.18) for the operator $L$ we get matrix representations

$$
L^{j}=\left(\begin{array}{ll}
L_{11}^{j} & L_{12}^{j} \\
L_{21}^{j} & L_{22}^{j}
\end{array}\right), \quad j=0,1,2
$$

for each operators $L^{j}, j=0,1,2$. Consequently, we can write $L_{22}^{-1}$ as

$$
L_{22}^{-1}=\left(L_{22}^{0}+L_{22}^{1}+L_{22}^{2}\right)^{-1}=\left(E_{\geq 2}+\left(L_{22}^{0}\right)^{-1} L_{22}^{1}+\left(L_{22}^{0}\right)^{-1} L_{22}^{2}\right)^{-1}\left(L_{22}^{0}\right)^{-1}
$$

with the identity operator $E_{\geq 2}$ acting in $\mathcal{L}_{\geq 2}$. Let us estimate now norms of the operators $\left(L_{22}^{0}\right)^{-1} L_{22}^{1}$ and $\left(L_{22}^{0}\right)^{-1} L_{22}^{2}$. 
The estimation of $\left\|\left(L_{22}^{0}\right)^{-1} L_{22}^{1}\right\| \| \quad$ Here we will follow the same lines as in [4]. It follows from (3.1), (4.4-4.5) that

$$
\begin{aligned}
& \left\|\left(L_{22}^{0}\right)^{-1} L_{22}^{1} G\right\|_{M} \\
& =z \sup _{\hat{\eta}_{1}}\left(( \frac { 1 } { 3 } ) ^ { | \eta _ { 1 } | } \sum _ { \hat { \gamma } _ { 1 } \subseteq \hat { \eta } _ { 1 } } \int _ { \Gamma _ { 0 } } \frac { ( | \eta _ { 1 } | + | \eta _ { 2 } | ) } { ( | \eta _ { 1 } | + | \eta _ { 2 } | ) } \operatorname { s u p } _ { \sigma _ { \eta _ { 2 } } } \left(\sum_{\hat{\gamma}_{2} \subseteq \hat{\eta}_{2}} \int\left|G\left(\hat{\gamma}_{1} \cup \hat{\gamma}_{2} \cup \hat{x}\right)\right|\right.\right. \\
& \left.\left.\times \prod_{\hat{y} \in\left(\hat{\eta}_{1} \backslash \hat{\gamma}_{1}\right) \cup\left(\hat{\eta}_{2} \backslash \hat{\gamma}_{2}\right)}\left|\left(e^{-\beta \varphi(x-y)\left(\sigma_{x}-\sigma_{y}\right)^{2}}-1\right)\right| \prod_{\hat{y} \in \hat{\gamma}_{1} \cup \hat{\gamma}_{2}} e^{-\beta \varphi(x-y)\left(\sigma_{x}-\sigma_{y}\right)^{2}} d \hat{x}\right) M^{\left|\eta_{2}\right|} d \eta_{2}\right) \\
& \leq z \sup _{\hat{\eta}_{1}}\left(\left(\frac{1}{3}\right)^{\left|\eta_{1}\right|} \sup _{\hat{x}}\left\{\sum_{\hat{\gamma}_{1} \subseteq \hat{\eta}_{1}} \prod_{\hat{y} \in \hat{\eta}_{1} \backslash \hat{\gamma}_{1}}\left|\left(e^{-\beta \varphi(x-y)\left(\sigma_{x}-\sigma_{y}\right)^{2}}-1\right)\right| \prod_{\hat{y} \in \hat{\gamma}_{1}} e^{-\beta \varphi(x-y)\left(\sigma_{x}-\sigma_{y}\right)^{2}}\right\}\right. \\
& \times \sup _{\hat{\gamma}_{1} \subseteq \hat{\eta}_{1}} \int \sup _{\sigma_{\eta_{2}}}\left(\sum_{\hat{\gamma}_{2} \subseteq \hat{\eta}_{2}} \int\left|G\left(\hat{\gamma}_{1} \cup \hat{\gamma}_{2} \cup \hat{x}\right)\right|\right. \text {. } \\
& \left.\left.\times \prod_{\hat{y} \in \hat{\eta}_{2} \backslash \hat{\gamma}_{2}}\left|\left(e^{-\beta \varphi(x-y)\left(\sigma_{x}-\sigma_{y}\right)^{2}}-1\right)\right| \prod_{\hat{y} \in \hat{\gamma}_{2}} e^{-\beta \varphi(x-y)\left(\sigma_{x}-\sigma_{y}\right)^{2}} d \hat{x}\right) M^{\left|\eta_{2}\right|} d \eta_{2}\right) .
\end{aligned}
$$

The inner sum in (4.8) for any $\hat{\eta}_{1}$ and any $\hat{x}$ equals to 1 :

$$
\begin{aligned}
& \sum_{\hat{\gamma}_{1} \subseteq \hat{\eta}_{1}} \prod_{\hat{y} \in \hat{\eta}_{1} \backslash \hat{\gamma}_{1}}\left|\left(e^{-\beta \varphi(x-y)\left(\sigma_{x}-\sigma_{y}\right)^{2}}-1\right)\right| \prod_{\hat{y} \in \hat{\gamma}_{1}} e^{-\beta \varphi(x-y)\left(\sigma_{x}-\sigma_{y}\right)^{2}} \\
& =\prod_{\hat{y} \in \hat{\eta}_{1}}\left(1-e^{-\beta \varphi(x-y)\left(\sigma_{x}-\sigma_{y}\right)^{2}}+e^{-\beta \varphi(x-y)\left(\sigma_{x}-\sigma_{y}\right)^{2}}\right)=1 .
\end{aligned}
$$

We use here the positivity of the potential $\varphi \geq 0$, so that

$$
\prod_{\hat{y} \in \hat{\gamma}_{2}} e^{-\beta \varphi(x-y)\left(\sigma_{x}-\sigma_{y}\right)^{2}} \leq 1,
$$

and

$$
\sup _{\sigma_{x}, \sigma_{y}}\left|\left(e^{-\beta \varphi(x-y)\left(\sigma_{x}-\sigma_{y}\right)^{2}}-1\right)\right|=1-e^{-4 \beta \varphi(x-y)} \equiv \kappa_{\beta}(x-y) .
$$

We will also use below the following inequality that holds for any non-negative $f(\gamma)$ :

$$
\sup _{\hat{\eta}}\left(\left(\frac{1}{3}\right)^{|\eta|}\left\{\sup _{\hat{\gamma} \subseteq \hat{\eta}} f(\hat{\gamma})\right\}\right) \leq \sup _{\hat{\eta}}\left(\left(\frac{1}{3}\right)^{|\eta|} f(\hat{\eta})\right) \text {. }
$$

Then we can continue (4.8) as follows:

$$
\begin{aligned}
& \leq z \sup _{\hat{\eta}_{1}}\left(\left(\frac{1}{3}\right)^{\left|\eta_{1}\right|} \sup _{\hat{\gamma}_{1} \subseteq \hat{\eta}_{1}} \iint \sup _{\sigma_{\eta_{2}}, \sigma_{x}}\left(\sum_{\hat{\gamma}_{2} \subseteq \hat{\eta}_{2}}\left|G\left(\hat{\gamma}_{1} \cup \hat{\gamma}_{2} \cup \hat{x}\right)\right|\right) \prod_{y \in \eta_{2} \backslash \gamma_{2}} \kappa_{\beta}(x-y) d x M^{\left|\eta_{2}\right|} d \eta_{2}\right) \\
& \leq z \sup _{\hat{\eta}_{1}}\left(\left(\frac{1}{3}\right)^{\left|\eta_{1}\right|} \iint \sum_{\gamma_{2} \subseteq \eta_{2}} \sup _{\sigma_{\gamma_{2}}, \sigma_{x}}\left|G\left(\hat{\eta}_{1} \cup \hat{\gamma}_{2} \cup \hat{x}\right)\right| \prod_{y \in \eta_{2} \backslash \gamma_{2}} \kappa_{\beta}(x-y) M^{\left|\eta_{2}\right|} d x d \eta_{2}\right)
\end{aligned}
$$




$$
\begin{aligned}
& =z \sup _{\hat{\eta}_{1}}\left(\left(\frac{1}{3}\right)^{\left|\eta_{1}\right|} \int_{\Gamma_{0}} \int_{\Gamma_{0}} \int_{R^{v}} \sup _{\sigma_{\gamma_{2}} ; \sigma_{x}}\left|G\left(\hat{\eta}_{1} \cup \hat{\gamma}_{2} \cup \hat{x}\right)\right| \prod_{y \in \gamma_{1}} \kappa_{\beta}(x-y) M^{\left|\gamma_{1}\right|} M^{\left|\gamma_{2}\right|} d \gamma_{1} d \gamma_{2} d x\right) \\
& =\frac{z}{M} e^{M C(\beta)} \sup _{\hat{\eta}_{1}}\left(\left(\frac{1}{3}\right)^{\left|\eta_{1}\right|} \int_{\Gamma_{0}}\left(\sup _{\sigma_{\tilde{\gamma}}}\left|G\left(\hat{\eta}_{1} \cup \hat{\tilde{\gamma}}\right)\right|\right)|\tilde{\gamma}| M^{|\tilde{\gamma}|} d \tilde{\gamma}\right) \leq \frac{z}{M} e^{M C(\beta)}\|G\|_{M}, \quad
\end{aligned}
$$

where $\hat{\tilde{\gamma}}=\hat{\gamma}_{2} \cup \hat{x}$. In the last step we use that for any $x$ :

$$
\begin{aligned}
& \int_{\Gamma_{0}} \prod_{y \in \gamma_{1}} \kappa_{\beta}(x-y) M^{\left|\gamma_{1}\right|} d \gamma_{1} \\
& \quad=1+\sum_{n=1}^{\infty} M^{n} \frac{1}{n !} \int_{R^{v}} \ldots \int_{R^{v}} \prod_{i=1}^{n} \kappa_{\beta}\left(y_{i}\right) d y_{1} \ldots d y_{n}=e^{M C(\beta)},
\end{aligned}
$$

with $C(\beta)=\int \kappa_{\beta}(y) d y$. Taking $M=\frac{1}{C(\beta)}$ we have

$$
\left\|\left(L_{22}^{0}\right)^{-1} L_{22}^{1}\right\|_{M} \leq \frac{z}{M} e^{M C(\beta)}=\varepsilon \cdot e
$$

The estimation of $\left\|\left(L_{22}^{0}\right)^{-1} L_{22}^{2}\right\|_{M} \quad$ Here we will use again relations (4.9), (4.10), (4.11) and (4.13).

$$
\begin{aligned}
& \left\|\left(L_{22}^{0}\right)^{-1} L_{22}^{2} F\right\|_{M} \\
& =\lambda \sup _{\hat{\eta}_{1}}\left(\left(\frac{1}{3}\right)^{\left|\eta_{1}\right|} \sum_{\hat{\gamma}_{1} \subseteq \hat{\eta}_{1}} \int_{\Gamma_{0}} \frac{\left(\left|\eta_{1}\right|+\left|\eta_{2}\right|\right)}{\left(\left|\eta_{1}\right|+\left|\eta_{2}\right|\right)} \sup _{\sigma_{\eta_{2}}} \mid \sum_{\hat{\gamma}_{2} \subseteq \hat{\eta}_{2}} \sum_{\hat{x} \in \hat{\gamma}_{1} \cup \hat{\gamma}_{2}}\left(F\left(\left(\hat{\gamma}_{1} \cup \hat{\gamma}_{2}\right)^{x}\right)-F\left(\hat{\gamma}_{1} \cup \hat{\gamma}_{2}\right)\right)\right. \\
& \left.\times \prod_{\hat{y} \in\left(\hat{\eta}_{1} \backslash \hat{\gamma}_{1}\right) \cup\left(\hat{\eta}_{2} \backslash \hat{\gamma}_{2}\right)}\left(e^{-\beta \varphi(x-y)\left(\sigma_{x}+\sigma_{y}\right)^{2}}-1\right) \prod_{\hat{y} \in\left(\hat{\gamma}_{1} \cup \hat{\gamma}_{2}\right) \backslash \hat{x}} e^{-\beta \varphi(x-y)\left(\sigma_{x}+\sigma_{y}\right)^{2}} \mid M^{\left|\eta_{2}\right|} d \eta_{2}\right) \\
& \leq \lambda \sup _{\hat{\eta}_{1}}\left(\left(\frac{1}{3}\right)^{\left|\eta_{1}\right|} \sup _{\hat{x} \in \hat{\eta}_{1}}\left\{\sum_{\hat{\gamma}_{1} \subseteq \hat{\eta}_{1} \backslash \hat{x}} \prod_{\hat{y} \in\left(\hat{\eta}_{1} \backslash \hat{x}\right) \backslash \hat{\gamma}_{1}}\left|\left(e^{-\beta \varphi(x-y)\left(\sigma_{x}-\sigma_{y}\right)^{2}}-1\right)\right| \prod_{\hat{y} \in \hat{\gamma}_{1}} e^{-\beta \varphi(x-y)\left(\sigma_{x}-\sigma_{y}\right)^{2}}\right\}\right. \\
& \times \sum_{\hat{x} \in \hat{\eta}_{1}} \sup _{\substack{\hat{\gamma}_{1} \subseteq \hat{\eta}_{1} \\
\hat{\gamma}_{1} \ni \hat{x}}} \int_{\Gamma_{0}} \sup _{\sigma_{\eta_{2}}}\left(\sum_{\hat{\gamma}_{2} \subseteq \hat{\eta}_{2}}\left|F\left(\left(\hat{\gamma}_{1} \cup \hat{\gamma}_{2}\right)^{x}\right)-F\left(\hat{\gamma}_{1} \cup \hat{\gamma}_{2}\right)\right|\right. \\
& \left.\left.\times \prod_{\hat{y} \in \hat{\gamma}_{2}} e^{-\beta \varphi(x-y)\left(\sigma_{x}+\sigma_{y}\right)^{2}} \prod_{\hat{y} \in \hat{\eta}_{2} \backslash \hat{\gamma}_{2}}\left|\left(e^{-\beta \varphi(x-y)\left(\sigma_{x}+\sigma_{y}\right)^{2}}-1\right)\right|\right) M^{\left|\eta_{2}\right|} d \eta_{2}\right) \\
& +\lambda \sup _{\hat{\eta}_{1}}\left(\left(\frac{1}{3}\right)^{\left|\eta_{1}\right|} \sup _{\hat{x} \in \hat{\eta}_{2}}\left\{\sum_{\hat{\gamma}_{1} \subseteq \hat{\eta}_{1}} \prod_{\hat{y} \in\left(\hat{\eta}_{1}\right) \backslash \hat{\gamma}_{1}}\left|\left(e^{-\beta \varphi(x-y)\left(\sigma_{x}-\sigma_{y}\right)^{2}}-1\right)\right| \prod_{\hat{y} \in \hat{\gamma}_{1}} e^{-\beta \varphi(x-y)\left(\sigma_{x}-\sigma_{y}\right)^{2}}\right\}\right. \\
& \times \sup _{\hat{\gamma}_{1} \subseteq \hat{\eta}_{1}} \int_{\Gamma_{0}} \sup _{\sigma_{\eta_{2}}}\left(\sum_{\hat{\gamma}_{2} \subseteq \hat{\eta}_{2}} \sum_{\hat{x} \in \hat{\gamma}_{2}}\left|F\left(\left(\hat{\gamma}_{1} \cup \hat{\gamma}_{2}\right)^{x}\right)-F\left(\hat{\gamma}_{1} \cup \hat{\gamma}_{2}\right)\right|\right. \\
& \left.\left.\times \prod_{\hat{y} \in \hat{\gamma}_{2} \backslash \hat{x}} e^{-\beta \varphi(x-y)\left(\sigma_{x}+\sigma_{y}\right)^{2}} \prod_{\hat{y} \in \hat{\eta}_{2} \backslash \hat{\gamma}_{2}}\left|\left(e^{-\beta \varphi(x-y)\left(\sigma_{x}+\sigma_{y}\right)^{2}}-1\right)\right|\right) M^{\left|\eta_{2}\right|} d \eta_{2}\right)
\end{aligned}
$$




$$
\begin{aligned}
& \leq \lambda \sup _{\hat{\eta}_{1}}\left(\left(\frac{1}{3}\right)^{\left|\eta_{1}\right|}\left|\eta_{1}\right| \sup _{\substack{\hat{\gamma}_{1} \subseteq \hat{\eta}_{1} \\
\hat{x} \in \hat{\gamma}_{1}}} \int_{\Gamma_{0}} \sum_{\gamma_{2} \subseteq \eta_{2}} \sup _{\sigma_{\gamma_{2}}} \mid\left(F\left(\left(\hat{\gamma}_{1} \cup \hat{\gamma}_{2}\right)^{x}\right)-F\left(\hat{\gamma}_{1} \cup \hat{\gamma}_{2}\right) \mid\right.\right. \\
& \left.\times \prod_{y \in \eta_{2} \backslash \gamma_{2}} \kappa_{\beta}(x-y) M^{\left|\eta_{2}\right|} d \eta_{2}\right) \\
& +\lambda \sup _{\hat{\eta}_{1}}\left(\left(\frac{1}{3}\right)^{\left|\eta_{1}\right|} \sup _{\hat{\gamma}_{1} \subseteq \hat{\eta}_{1}} \int_{\Gamma_{0}} \sum_{\gamma_{2} \subseteq \eta_{2}} \sup _{\sigma_{\gamma_{2}}} \sum_{\hat{x} \in \hat{\gamma}_{2}}\left|F\left(\left(\hat{\gamma}_{1} \cup \hat{\gamma}_{2}\right)^{x}\right)-F\left(\hat{\gamma}_{1} \cup \hat{\gamma}_{2}\right)\right|\right. \\
& \left.\times \prod_{y \in \eta_{2} \backslash \gamma_{2}} \kappa_{\beta}(x-y) M^{\left|\eta_{2}\right|} d \eta_{2}\right) \\
& \leq 2 \lambda \sup _{\hat{\eta}_{1}}\left(\left(\frac{1}{3}\right)^{\left|\eta_{1}\right|}\left|\eta_{1}\right| \int_{\Gamma_{0}} \sup _{\sigma_{\gamma_{2}}}\left|F\left(\hat{\eta}_{1} \cup \hat{\gamma}_{2}\right)\right| M^{\left|\gamma_{2}\right|} d \gamma_{2}\right) \int_{\Gamma_{0}} \prod_{u \in \xi} \kappa_{\beta}(u) M^{|\xi|} d \xi \\
& +\lambda e \sup _{\hat{\eta}_{1}}\left(\left(\frac{1}{3}\right)^{\left|\eta_{1}\right|} \int_{\Gamma_{0}} \sup _{\sigma_{\gamma_{2}}}\left(\sum_{\hat{x} \in \hat{\gamma}_{2}}\left|\left(F\left(\left(\hat{\eta}_{1} \cup \hat{\gamma}_{2}\right)^{x}\right)-F\left(\hat{\eta}_{1} \cup \hat{\gamma}_{2}\right)\right)\right|\right) M^{\left|\gamma_{2}\right|} d \gamma_{2}\right) \\
& \leq 2 \lambda e \sup _{\hat{\eta}_{1}}\left(\left(\frac{1}{3}\right)^{\left|\eta_{1}\right|} \int_{\Gamma_{0}}\left(\left|\eta_{1}\right|+\left|\gamma_{2}\right|\right) \sup _{\sigma_{\gamma_{2}}}\left|F\left(\hat{\eta}_{1} \cup \hat{\gamma}_{2}\right)\right| M^{\left|\gamma_{2}\right|} d \gamma_{2}\right)=2 \lambda e\|F\|_{M} .
\end{aligned}
$$

It follows from (4.4) that

$$
\text { || }\left(L_{22}^{0}\right)^{-1} \mid \|_{M} \leq \frac{1}{2},
$$

then from (4.7) and (4.14), (4.15) we finally have

$$
\left\|\left(L_{22}\right)^{-1} \mid\right\|_{M} \leq \frac{1}{2(1-(e \varepsilon+2 e \lambda))}<\frac{1}{2}(1+3 \varepsilon+6 \lambda)
$$

for all small enough $\varepsilon$ and $\lambda$. Lemma 3.4 is proved.

\subsection{Proof of Lemma 3.5}

\subsubsection{Operator $L_{11}$}

Functions $G \in \mathcal{L}_{\leq 1}$ have the form:

$$
G(\hat{\eta})= \begin{cases}G_{0}, & \eta=\emptyset \\ G_{1}(\hat{x}), & |\eta|=1 \\ 0, & |\eta| \geq 2\end{cases}
$$

and

$$
\|G\|_{M}=\sup \left\{\frac{1}{3} \sup _{\hat{x}}\left|G_{1}(\hat{x})\right| ; M \int \sup _{\sigma_{x}}\left|G_{1}(\hat{x})\right| d x\right\}+\left|G_{0}\right| .
$$


The function $L_{11} G$ by (2.14) has the following components:

$$
\begin{gathered}
\left(L_{11} G\right)_{0}=z \int G_{1}(\hat{y}) d \hat{y}, \\
\left(L_{11} G\right)_{1}(\hat{x})=-G_{1}(\hat{x})+z\left(\int G_{1}(\hat{y})\left(e^{-\beta \varphi(x-y)\left(\sigma_{x}-\sigma_{y}\right)^{2}}-1\right) d \hat{y}\right)(\hat{x}) \\
+\lambda\left(G_{1}\left(\hat{x}^{\star}\right)-G_{1}(\hat{x})\right),
\end{gathered}
$$

with $\hat{x}^{\star}=\left(x,-\sigma_{x}\right)$. Then using the estimates $\kappa_{\beta}(x-y)=1-e^{-4 \beta \varphi(x-y)} \leq 1$, we have:

$$
\begin{aligned}
& \left\|L_{11} G\right\|_{M}=\sup _{\hat{\eta}}\left(\left(\frac{1}{3}\right)^{|\eta|} \int_{\Gamma_{0}} \sup _{\substack{\sigma_{\xi}: \\
\hat{\eta} \cup \hat{\xi}=\hat{x}}}\left|\left(L_{11} G\right)_{1}(\hat{\eta} \cup \hat{\xi})\right| M^{|\xi|} d \xi\right)+\left|\left(L_{11} G\right)_{0}\right| \\
& \leq \sup \left\{\frac { 1 } { 3 } \operatorname { s u p } _ { \hat { x } } \left((1+\lambda)\left|G_{1}(\hat{x})\right|+\lambda\left|G_{1}\left(\hat{x}^{\star}\right)\right|\right.\right. \\
& \left.+z \int\left|G_{1}(\hat{y})\right| \cdot\left|\left(e^{-\beta \varphi(x-y)\left(\sigma_{x}-\sigma_{y}\right)^{2}}-1\right)\right| d \hat{y}\right) ; \\
& \int \sup _{\sigma_{x}}\left((1+\lambda)\left|G_{1}(\hat{x})\right|+\lambda\left|G_{1}\left(\hat{x}^{\star}\right)\right|\right. \\
& \left.\left.+z \int\left|G_{1}(\hat{y})\right| \cdot\left|\left(e^{-\beta \varphi(x-y)\left(\sigma_{x}-\sigma_{y}\right)^{2}}-1\right)\right| d \hat{y}\right) M d x\right\} \\
& +\frac{z}{M} \int\left|G_{1}(\hat{y})\right| M d \hat{y} \\
& \leq \sup \left\{\frac{1+2 \lambda+\varepsilon}{3} \sup _{\hat{x}}\left|G_{1}(\hat{x})\right| ;(1+2 \lambda+2 \varepsilon) \int \sup _{\sigma_{x}}\left|G_{1}(\hat{x})\right| M d x\right\} \\
& \leq(1+2 \lambda+2 \varepsilon)\|G\|_{M} \text {. }
\end{aligned}
$$

Thus,

$$
\left\|L_{11}\right\|_{M} \leq 1+2 \lambda+2 \varepsilon .
$$

\subsubsection{Operator $L_{12}$}

The operator $L_{12}: \mathcal{L}_{\geq 2} \rightarrow \mathcal{L}_{\leq 1}$ has the following components:

$$
\left(L_{12} G\right)_{0}=0, \quad\left(L_{12} G\right)_{1}(\hat{x})=z \int G_{2}(\hat{x} \cup \hat{y}) e^{-\beta \varphi(x-y)\left(\sigma_{x}-\sigma_{y}\right)^{2}} d \hat{y},
$$

where $G_{2} \in \mathcal{L}_{2}$ is a two-spin configuration component of $G \in \mathcal{L}_{\geq 2}$. Then

$$
\begin{gathered}
\left\|G_{2}\right\|_{M}=\sup \left\{\frac{2}{9} \sup _{\hat{x} \cup \hat{y}}\left|G_{2}(\hat{x} \cup \hat{y})\right| ; \frac{2}{3} \sup _{\hat{x}} \int \sup _{\sigma_{y}}\left|G_{2}(\hat{x} \cup \hat{y})\right| M d y ;\right. \\
\left.\iint \sup _{\sigma_{x} ; \sigma_{y}}\left|G_{2}(\hat{x} \cup \hat{y})\right| M^{2} d x d y\right\} \leq\|G\|_{M},
\end{gathered}
$$


and hence,

$$
\begin{aligned}
\left\|L_{12} G\right\|_{M}= & \left\|\left(L_{12} G\right)_{1}\right\|_{M} \\
= & \sup \left\{\frac{z}{3} \sup _{\hat{x}}\left|\int G_{2}(\hat{x} \cup \hat{y}) e^{-\beta \varphi(x-y)\left(\sigma_{x}-\sigma_{y}\right)^{2}} d \hat{y}\right| ;\right. \\
& \left.\quad z \int \sup _{\sigma_{x}}\left|\int G_{2}(\hat{x} \cup \hat{y}) e^{-\beta \varphi(x-y)\left(\sigma_{x}-\sigma_{y}\right)^{2}} d \hat{y}\right| M d x\right\} \\
\leq & \frac{z}{M} \sup \left\{\frac{1}{3} \sup _{\hat{x}} \int \sup _{\sigma_{y}}\left|G_{2}(\hat{x} \cup \hat{y})\right| M d y ; \iint \sup _{\sigma_{x} ; \sigma_{y}}\left|G_{2}(\hat{x} \cup \hat{y})\right| M^{2} d x d y\right\} \\
\leq & \varepsilon\left\|G_{2}\right\|_{M} .
\end{aligned}
$$

Thus (4.18) implies

$$
\left\|L_{12}\right\|_{M} \leq \varepsilon
$$

\subsubsection{Operator $L_{21}$}

Using again representation (4.16) for the function $G=\left(G_{0}, G_{1}\right) \in \mathcal{L}_{\leq 1}$, we get

$$
\begin{aligned}
\left(L_{21} G\right)(\hat{\eta})= & \left(L_{21} G_{1}\right)(\hat{\eta}) \\
= & z \int G_{1}(\hat{x}) \prod_{\hat{y} \in \hat{\eta}}\left(e^{-\beta \varphi(x-y)\left(\sigma_{x}-\sigma_{y}\right)^{2}}-1\right) d \hat{x} \\
& +\lambda \sum_{\hat{x} \in \hat{\eta}}\left(G_{1}\left(\hat{x}^{\star}\right)-G_{1}(\hat{x})\right) \prod_{\hat{y} \in \hat{\eta} \hat{x}}\left(e^{-\beta \varphi(x-y)\left(\sigma_{x}+\sigma_{y}\right)^{2}}-1\right), \quad|\eta| \geq 2 .
\end{aligned}
$$

We denote the first term in (4.19) by $\Phi_{1}(\hat{\eta})$ and the second one by $\Phi_{2}(\hat{\eta}),|\eta| \geq 2$. The first term $\Phi_{1}(\hat{\eta})$ can be estimated in a similar way as in [4]. Using the equality $M C(\beta)=1$ and estimates (4.10-4.11) we have:

$$
\begin{aligned}
\left\|\Phi_{1}\right\|_{M}= & z \sup _{\hat{\eta}}\left(\left(\frac{1}{3}\right)^{|\eta|} \int_{\Gamma_{0}}(|\eta|+|\xi|)\right. \\
& \left.\times \sup _{\sigma_{\xi}}\left|\int G_{1}(\hat{x}) \prod_{\hat{y} \in \hat{\eta} \cup \hat{\xi}}\left(e^{-\beta \varphi(x-y)\left(\sigma_{x}-\sigma_{y}\right)^{2}}-1\right) d \hat{x}\right| M^{|\xi|} d \xi\right) \\
\leq & z \int\left|G_{1}(\hat{x})\right| d \hat{x} \\
& \times \sup _{\hat{\eta}, \hat{x}}\left(\left(\frac{1}{3}\right)^{|\eta|} \int_{\Gamma_{0}}(|\eta|+|\xi|) \sup _{\sigma_{\xi}} \prod_{\hat{y} \in \hat{\eta} \cup \hat{\xi}}\left|\left(e^{-\beta \varphi(x-y)\left(\sigma_{x}-\sigma_{y}\right)^{2}}-1\right)\right| M^{|\xi|} d \xi\right) \\
\leq & \frac{z}{M} \int_{\sigma_{x}} \sup _{\sigma_{x}}\left|G_{1}(\hat{x})\right| M d x \cdot \sup _{n: n+|\xi| \geq 2}\left(\left(\frac{1}{3}\right)^{n} \int(n+|\xi|) \prod_{u \in \xi} \kappa_{\beta}(u) M^{|\xi|} d \xi\right) \\
\leq & \varepsilon\left\|G_{1}\right\|_{M} \int \sup _{n: n+|\xi| \geq 2}\left(\left(\frac{1}{3}\right)^{n}(n+|\xi|) \prod_{u \in \xi} \kappa_{\beta}(u) M^{|\xi|} d \xi\right)
\end{aligned}
$$




$$
\begin{aligned}
\leq & \varepsilon\left\|G_{1}\right\|_{M}\left(\sup _{n \geq 2}\left\{\left(\frac{1}{3}\right)^{n} n\right\}+C(\beta) M \sup _{n \geq 1}\left\{\left(\frac{1}{3}\right)^{n}(n+1)\right\}\right. \\
& \left.+\sum_{k \geq 2} \sup _{n \geq 0}\left\{\left(\frac{1}{3}\right)^{n}(n+k)\right\} \frac{(C(\beta) M)^{k}}{k !}\right) \\
\leq & \varepsilon\left\|G_{1}\right\|_{M}\left(\frac{2}{9}+\frac{2}{3} C(\beta) M+\sum_{k=2}^{\infty} k \frac{(C(\beta) M)^{k}}{k !}\right) \leq 4 \varepsilon\left\|G_{1}\right\|_{M} .
\end{aligned}
$$

For the second term we have the following estimate:

$$
\begin{aligned}
& \left\|\Phi_{2}\right\|_{M} \leq \lambda \sup _{\hat{\eta}}\left(( \frac { 1 } { 3 } ) ^ { | \eta | } \int _ { \Gamma _ { 0 } } ( | \eta | + | \xi | ) \operatorname { s u p } _ { \substack { \sigma _ { \xi } : \\
| \eta | + | \xi | \geq 2 } } \left(\sum_{\hat{x} \in \hat{\eta} \cup \hat{\xi}}\left|G_{1}\left(\hat{x}^{\star}\right)-G_{1}(\hat{x})\right|\right.\right. \\
& \left.\left.\times \prod_{\hat{y} \in \hat{\eta} \backslash \hat{x}}\left|\left(e^{-\beta \varphi(x-y)\left(\sigma_{x}+\sigma_{y}\right)^{2}}-1\right)\right| \prod_{\hat{y} \in \hat{\xi} \backslash \hat{x}}\left|\left(e^{-\beta \varphi(x-y)\left(\sigma_{x}+\sigma_{y}\right)^{2}}-1\right)\right|\right) M^{|\xi|} d \xi\right) \\
& \leq 2 \lambda \sup _{\hat{x}}\left|G_{1}(\hat{x})\right| \sup _{\hat{\eta}, \hat{x} \in \hat{\eta}}\left(\left(\frac{1}{3}\right)^{|\eta|}|\eta| \int_{\Gamma_{0}}(|\eta|+|\xi|)\right. \\
& \left.\times \sup _{\sigma_{\xi}} \prod_{\hat{y} \in \hat{\xi}}\left|\left(e^{-\beta \varphi(x-y)\left(\sigma_{x}+\sigma_{y}\right)^{2}}-1\right)\right| M^{|\xi|} d \xi\right) \\
& +\lambda \sup _{\hat{\eta}}\left(\left(\frac{1}{3}\right)^{|\eta|} \int_{\Gamma_{0}}(|\eta|+|\xi|)\right. \text {. } \\
& \left.\times \sup _{\sigma_{\xi}}\left(\sum_{\hat{x} \in \hat{\xi}}\left|G_{1}\left(\hat{x}^{\star}\right)-G_{1}(\hat{x})\right| \prod_{\hat{y} \in \hat{\xi} \backslash \hat{x}}\left|\left(e^{-\beta \varphi(x-y)\left(\sigma_{x}+\sigma_{y}\right)^{2}}-1\right)\right|\right) M^{|\xi|} d \xi\right) \\
& \leq 2 \lambda \sup _{\hat{x}}\left|G_{1}(\hat{x})\right| \int_{\Gamma_{0}} \sup _{n: n+|\xi| \geq 2}\left(\left(\frac{1}{3}\right)^{n} n(n+|\xi|)\right) \prod_{y \in \xi} \kappa_{\beta}(y) M^{|\xi|} d \xi \\
& +2 \lambda \int \sup _{\sigma_{x}}\left|G_{1}(\hat{x})\right| M d x \int_{\Gamma_{0}} \sup _{n: n+|\tilde{\xi}| \geq 1}\left(\left(\frac{1}{3}\right)^{n}(n+1+|\tilde{\xi}|)\right) \prod_{u \in \tilde{\xi}} \kappa_{\beta}(u) M^{|\tilde{\xi}|} d \tilde{\xi} \\
& \leq 24 \lambda\left(\frac{1}{3} \sup _{\hat{x}}\left|G_{1}(\hat{x})\right|\right)+12 \lambda \int \sup _{\sigma_{x}}\left|G_{1}(\hat{x})\right| M d x \leq 36 \lambda\left\|G_{1}\right\|_{M} .
\end{aligned}
$$

Here we use that

$$
\begin{gathered}
\sup _{n \geq 2}\left(\left(\frac{1}{3}\right)^{n} n^{2}\right)+C(\beta) M \sup _{n \geq 1}\left(\left(\frac{1}{3}\right)^{n} n(n+1)\right) \\
+\sum_{k \geq 2} \frac{(C(\beta) M)^{k}}{k !} \sup _{n \geq 0}\left(\left(\frac{1}{3}\right)^{n} n(n+k)\right) \leq 4, \\
\sup _{n \geq 1}\left(\left(\frac{1}{3}\right)^{n}(n+1)\right)+\sum_{k \geq 1} \frac{(C(\beta) M)^{k}}{(k) !} \sup _{n \geq 0}\left(\left(\frac{1}{3}\right)^{n}(n+k+1)\right) \leq 6 .
\end{gathered}
$$


Finally,

$$
\left\|L_{21}\right\|_{M} \leq 4 \varepsilon+36 \lambda .
$$

Lemma 3.5. is proved completely.

\subsection{Proof of Lemma 3.6}

Using (3.8) with (3.21-3.24) we have for all small enough $\varepsilon$ and $\lambda$ :

$$
\begin{aligned}
\|\| \mathcal{F}(S)\|\|_{M} \leq & \left\|L_{22}^{-1}\left|\left\|_{M}\right\|\right| L_{21}\right\| \|_{M} \\
& +\left\|L_{22}^{-1}\right\|\left\|_{M}\right\| L_{11}\|\|_{M}\|S\|\left\|_{M}+\right\| L_{22}^{-1}\|\|_{M}\left\|L_{12}\right\|\left\|_{M}\right\| S\|\|_{M}^{2} \\
\leq & \frac{1}{2}(1+3 \varepsilon+6 \lambda)(4 \varepsilon+36 \lambda)+\frac{1}{2}(1+3 \varepsilon+6 \lambda)(1+2 \varepsilon+2 \lambda)(8 \varepsilon+48 \lambda) \\
& +\frac{1}{2}(1+3 \varepsilon+6 \lambda) \varepsilon(8 \varepsilon+48 \lambda)^{2}<8 \varepsilon+48 \lambda,
\end{aligned}
$$

what proves the inclusion (3.25).

Further,

$$
\begin{aligned}
\mathcal{F}\left(S_{1}\right)-\mathcal{F}\left(S_{2}\right)= & L_{22}^{-1}\left(S_{1}-S_{2}\right) L_{11}+L_{22}^{-1}\left(S_{1}-S_{2}\right) L_{12} S_{1} \\
& +L_{22}^{-1} S_{2} L_{12}\left(S_{1}-S_{2}\right)
\end{aligned}
$$

consequently, using again (3.21-3.24) we have for any $S_{1}, S_{2} \in \mathcal{B}_{8 \varepsilon}$

$$
\begin{aligned}
& \|\| \mathcal{F}\left(S_{1}\right)-\mathcal{F}\left(S_{2}\right) \|_{M} \\
& \quad \leq\left(\frac{1}{2}(1+3 \varepsilon+6 \lambda)(1+2 \varepsilon+2 \lambda)+(1+3 \varepsilon+6 \lambda)(8 \varepsilon+48 \lambda) \varepsilon\right)\left\|\mid S_{1}-S_{2}\right\|_{M} .
\end{aligned}
$$

Since for small enough $\varepsilon$ and $\lambda$ :

$$
\begin{aligned}
c & =\frac{1}{2}(1+3 \varepsilon+6 \lambda)(1+2 \varepsilon+2 \lambda)+(1+3 \varepsilon+6 \lambda)(8 \varepsilon+48 \lambda) \varepsilon \\
& =\frac{1}{2}+O(\varepsilon)+O(\lambda)<1,
\end{aligned}
$$

the inequality (3.26) is proved.

\subsection{Proof of Lemma 3.7}

To prove (3.30) we have to find for any $G \in \mathcal{L}$ functions $g_{\leq 1} \in \mathcal{L}_{\leq 1}$ and $g_{\geq 2} \in \mathcal{L}_{\geq 2}$, such that

$$
G=\left(g_{\leq 1}+S g_{\leq 1}\right)+\left(g_{\geq 2}+T g_{\geq 2}\right),
$$

and to prove that the decomposition (4.21) is unique. The decomposition (4.21) is equivalent to the following relations

$$
g_{\leq 1}+T g_{\geq 2}=G_{\leq 1}, g_{\geq 2}+S g_{\leq 1}=G_{\geq 2}
$$


where $G_{\leq 1} \in \mathcal{L}_{\leq 1}$ and $G_{\geq 2} \in \mathcal{L}_{\geq 2}$ are the components of the function $G=\left(G_{\leq 1}, G_{\geq 2}\right) \in \mathcal{L}$. Then (4.22) implies that

$$
G_{\leq 1}-T G_{\geq 2}=g_{\leq 1}-T S g_{\leq 1}
$$

consequently,

$$
g_{\leq 1}=\left(E_{\leq 1}-T S\right)^{-1}\left(G_{\leq 1}-T G_{\geq 2}\right)
$$

and analogously,

$$
g_{\geq 2}=\left(E_{\geq 2}-S T\right)^{-1}\left(G_{\geq 2}-S G_{\leq 1}\right) .
$$

By (3.27), ( 3.29) for small enough $\varepsilon$ and $\lambda$ the operators $T S$ in $\mathcal{L}_{\leq 1}$ and $S T$ in $\mathcal{L}_{\geq 2}$ have small norms, consequently the functions $g_{\leq 1}, g_{\geq 2}$ are unique defined. Lemma is proved.

\subsection{Proof of Lemma 3.8}

According to the general scheme, see (3.9) and (3.10), the operator $L_{2}$ can be represented in the following form

$$
\begin{aligned}
& L_{2}=P_{\geq 2}^{-1}\left(L_{22}+L_{21} T\right) P_{\geq 2}, \\
& P_{\geq 2}: \hat{\mathcal{L}}_{\geq 2} \rightarrow \mathcal{L}_{\geq 2}, \quad P_{\geq 2}^{-1}: \mathcal{L}_{\geq 2} \rightarrow \hat{\mathcal{L}}_{\geq 2},
\end{aligned}
$$

and an analogous representation holds for the inverse operator $L_{2}^{-1}$ :

$$
L_{2}^{-1}=P_{\geq 2}^{-1}\left(L_{22}+L_{21} T\right)^{-1} P_{\geq 2} .
$$

Since

$$
\left(L_{22}+L_{21} T\right)^{-1}=\left(E_{\geq 2}+L_{22}^{-1} L_{21} T\right)^{-1} L_{22}^{-1},
$$

then using estimates (3.21), (3.24), (3.29) we have for small $\varepsilon$ and $\lambda$

$$
\left\|L_{22}^{-1} L_{21} T\right\|_{M}<(4 \varepsilon+36 \lambda)^{2}(1+O(\varepsilon)+O(\lambda)),
$$

and consequently,

$$
\left\|\mid\left(L_{22}+L_{21} T\right)^{-1}\right\|_{M}<\frac{1}{2}(1+5 \varepsilon+8 \lambda)
$$

for small enough $\varepsilon$ and $\lambda$. Estimate (3.29) on the norm of $T$ imply that

$$
\left\|\left.\left|P_{\geq 2}\right|\right|_{M} \leq 1, \quad\right\| P_{\geq 2}^{-1} \mid \|_{M} \leq 1+8 \varepsilon+48 \lambda .
$$

Finally the estimate (3.31) follows from (4.24), (4.25) and (4.26). Lemma is proved.

\subsection{Proof of Lemma 3.9}

Using our constructions above we obtain that the subspace $\hat{\mathcal{H}}_{1}=\overline{\hat{\mathcal{L}}_{1}}$ is invariant for the operator $L$, so that the restriction $\left.L\right|_{\mathcal{H}_{1}}$ is a bounded self-adjoint operator in $\mathcal{H}_{1}$. form

Analysis of the operator $L_{11}$, see (4.17), shows that the operator $L_{11}^{\prime}$, acting in $\mathcal{L}_{1}$ has a

$$
\begin{aligned}
\left(L_{11}^{\prime} G_{1}\right)(\hat{x})= & -G_{1}(\hat{x})+z \int G_{1}(\hat{y})\left(e^{-\beta \varphi(x-y)\left(\sigma_{x}-\sigma_{y}\right)^{2}}-1\right) d \hat{y} \\
& +\lambda\left(G_{1}\left(\hat{x}^{\star}\right)-G_{1}(\hat{x})\right), \quad G_{1} \in \mathcal{L}_{1} .
\end{aligned}
$$


Now (3.23), (3.27) imply that the operator $\left.L_{12} S\right|_{\mathcal{L}_{1}}$ has a small norm:

$$
\left\|\mid L_{12}\left(\left.S\right|_{\mathcal{L}_{1}}\right)\right\|_{M} \leq(8 \varepsilon+48 \lambda) \varepsilon
$$

hence, the operator $L_{11}^{\prime}+L_{12}\left(\left.S\right|_{\mathcal{L}_{1}}\right)$ can be rewritten as

$$
L_{11}^{\prime}+L_{12}\left(\left.S\right|_{\mathcal{L}_{1}}\right)=-E+R
$$

where

$\left(R G_{1}\right)(\hat{x})=z \int G_{1}(\hat{y})\left(e^{-\beta \varphi(x-y)\left(\sigma_{x}-\sigma_{y}\right)^{2}}-1\right) d \hat{y}+\lambda\left(G_{1}\left(\hat{x}^{\star}\right)-G_{1}(\hat{x})\right)+L_{12}\left(\left.S\right|_{\mathcal{L}_{1}}\right) G_{1}(\hat{x})$,

and

$$
\|R\|_{M} \leq \varepsilon+2 \lambda+(8 \varepsilon+48 \lambda) \varepsilon<2 \varepsilon+3 \lambda
$$

for small enough $\varepsilon$ and $\lambda$. Using the estimates on the norms of the operators $P_{1}$ and $P_{1}^{-1}$, see (3.33-3.35):

$$
\left\|\left|P_{1}\right|\right\|_{M} \leq 1, \quad\left\||| P_{1}^{-1}\right\|_{M} \leq 1+17 \varepsilon+96 \lambda,
$$

we have for small enough $\varepsilon$ and $\lambda$

$$
\begin{aligned}
\left\|L_{1}+E_{\hat{\mathcal{L}}_{1}}\right\|_{M} & =\left\|P_{1}^{-1} R P_{1}\right\|_{M} \leq\|R\|_{M} \cdot\left\|P_{1}^{-1}\right\|_{M} \\
& \leq(2 \varepsilon+3 \lambda)(1+17 \varepsilon+96 \lambda)<3 \varepsilon+4 \lambda .
\end{aligned}
$$

Finally, the proposition (3.11) implies that

$$
\left\|L_{1}+E_{\hat{\mathcal{H}}_{1}}\right\|_{\mathcal{H}} \leq\left\||| L_{1}+E_{\hat{\mathcal{L}}_{1}}\right\|_{M} \leq 3 \varepsilon+4 \lambda,
$$

that gives the position for the spectrum $\sigma_{1}$ in (2.18) with $\gamma_{1}=3 \varepsilon+4 \lambda$.

Applying the similar reasoning to the operator $\left(\left.L\right|_{\hat{\mathcal{H}} \geq 2}\right)^{-1}$ in the invariant subspace $\hat{\mathcal{H}}_{\geq 2}$ together with the estimate (3.31) we obtain that under small enough $\varepsilon$ and $\lambda$ the spectrum $\sigma_{2}$ of the operator $L_{2}$ is bounded from above by the value $-2+\gamma_{2}$ with $\gamma_{2}=30 \varepsilon+120 \lambda$. Thus, we proved the inclusions (2.18).

The last step is to prove the decomposition (2.17). Since for small enough $\varepsilon$ and $\lambda$ the spectra $\sigma_{0}, \sigma_{1}, \sigma_{2}$ are not overlapping the subspaces $\mathcal{H}_{0}, \mathcal{H}_{1}, \mathcal{H}_{\geq 2}$ are mutually orthogonal. Let us prove that the sum (2.17) gives a complete decomposition of the space $\mathcal{H}$. We know that according to the decomposition (3.36) any function $G \in \mathcal{L}$ has a representation of the form

$$
G=G_{0}+G_{1}+G_{\geq 2}, \quad G_{0} \in \mathcal{L}_{0}, G_{1} \in \hat{\mathcal{L}}_{1}, G_{\geq 2} \in \hat{\mathcal{L}}_{\geq 2}
$$

Moreover, any component of the decomposition (4.27) equals to the orthogonal projection of $G$ to the corresponding invariant subspace

$$
G_{0}=P_{\mathcal{H}_{0}} G, \quad G_{1}=P_{\mathcal{H}_{1}} G, \quad G_{\geq 2}=P_{\mathcal{H}_{\geq 2}} G,
$$

so that all vectors $G_{0}, G_{1}, G_{\geq 2}$ are mutually orthogonal and

$$
\|G\|_{\mathcal{H}}^{2}=\left\|P_{\mathcal{H}_{0}} G\right\|_{\mathcal{H}}^{2}+\left\|P_{\mathcal{H}_{1}} G\right\|_{\mathcal{H}}^{2}+\left\|P_{\mathcal{H}_{\geq 2}} G\right\|_{\mathcal{H}}^{2} .
$$

This equality holds for a dense set $\mathcal{L}$ in $\mathcal{H}$, consequently it is true for any element from $\mathcal{H}$, what is equivalent to the decomposition (2.17). Lemma 3.9 is completely proved. 


\section{References}

1. Angelescu, N., Minlos, R.A., Zagrebnov, V.A.: The lower spectral branch of the generator of the stochastic dynamics for the classical Heisenberg model. In: Minlos, R.A., Shlosman, S., Suhov, Yu.M. (eds.) On Dobrushin's Way: From Probability Theory to Statistical Physics. Amer. Math. Soc. Transl. (2), vol. 198, pp. 1-11 (2000)

2. Bertini, L., Cancrini, N., Cesi, F.: The spectral gap for a Glauber-type dynamics in a continuous gas. Ann. Inst. H. Poincaré Probab. Stat. 38, 91-108 (2002)

3. Kondratiev, Yu.G., Minlos, R.A.: One-particle subspaces in the stochastic XY model. J. Stat. Phys. 87(3/4), 613-642 (1997)

4. Kondratiev, Yu., Minlos, R., Zhizhina, E.: One-particle subspace of the Glauber dynamics generator for continuous particle systems. Rev. Math. Phys. 16(9), 1073-1114 (2004)

5. Kondratiev, Yu.G., Kuna, T.: Harmonic analysis on configuration spaces, I: general theory. Infin. Dimens. Anal. Quantum Probab. Relat. Top. 5, 201-233 (2002)

6. Kondratiev, Yu., Lytvynov, E.: Glauber dynamics of continuous particle systems. Ann. Inst. H. Poincare, Ser. B 41, 685-702 (2005)

7. Malyshev, V.A., Minlos, R.A.: Linear Infinite-Particle Operators. American Mathematical Society, Providence (1995)

8. Minlos, R.A.: Invariant subspaces of Ising stochastic dynamics (for small $\beta$ ). Markov Process. Relat. Fields 2(2), 263-284 (1996)

9. Minlos, R.A., Suhov, Yu.M.: On the spectrum of the generator of an infinite system of interacting diffusions. Commun. Math. Phys. 206, 463-489 (1999)

10. Minlos, R.A.: Lectures on statistical physics. Usp. Mat. Nauk 1, 133-190 (1968)

11. Ruelle, D.: Statistical Mechanics. Rigorous Results. Benjamins, Amsterdam (1969)

12. Ruelle, D.: Superstable interaction in classical statistical mechanics. Commun. Math. Phys. 18, 127-159 (1970)

13. Wu, L.: Estimate of spectral gap for continuous gas. Ann. Inst. H. Poincare PR 40, 387-409 (2004)

14. Zhizhina, E.: Convergence properties of quasi-particles of various species in the stochastic Blume-Capel model. Markov Process. Relat. Fields 10(2), 307-326 (2004) 